\title{
The legacy of maternal SARS-CoV-2 infection on the immunology of the neonate
}

\author{
Sarah Gee $\odot^{1}$, Manju Chandiramani ${ }^{2}$, Jeffrey Seow $\odot^{3}$, Emily Pollock $\odot^{1}$, Carlotta Modestini ${ }^{2}$, \\ Abhishek Das', Timothy Tree', Katie J. Doores ${ }^{\left({ }^{3}\right.}{ }^{3}$, Rachel M. Tribe ${ }^{4}{ }^{4}$ and Deena L. Gibbons ${ }^{1}{ }^{1 凶}$
}

\begin{abstract}
Despite extensive studies into severe acute respiratory syndrome coronavirus 2 (SARS-CoV-2), the effect of maternal infection on the neonate is unclear. To investigate this, we characterized the immunology of neonates born to mothers with confirmed SARS-CoV-2 infection during pregnancy. Here we show that maternal SARS-CoV-2 infection affects the neonatal immune system. Despite similar proportions of $B$ cells, $\mathrm{CD4}^{+} \mathbf{T}$ cells and $\mathrm{CD8}^{+} \mathbf{T}$ cells, increased percentages of natural killer cells, ${\mathrm{V} \delta 2^{+}}^{+} \delta$ $T$ cells and regulatory $T$ cells were detected in neonates born to mothers with recent or ongoing infection compared with those born to recovered or uninfected mothers. Increased plasma cytokine levels were also evident in neonates and mothers within the recent or ongoing infection group. Cytokine functionality was enhanced in neonates born to SARS-CoV-2-exposed mothers, compared to those born to uninfected mothers. In most neonates, this immune imprinting was nonspecific, suggesting vertical transmission of SARS-CoV-2 is limited, a finding supported by a lack of SARS-CoV-2-specific IgM in neonates despite maternal IgG transfer.
\end{abstract}

( espite the ongoing coronavirus disease 2019 (COVID-19) pandemic, the effect of maternal SARS-CoV-2 infection on the immunology of the developing neonate is unclear. SARS-CoV-2 infection in pregnancy has been reported to lead to variable outcomes for the mother. The majority of infected pregnant women are asymptomatic or experience mild symptoms ${ }^{1}$. Nonetheless, pregnant women with SARS-CoV-2, particularly in the third trimester, are at increased risk for hospitalization and intensive care $^{2}$, and existing evidence demonstrates that rates of maternal infection increased in the second wave ${ }^{3}$. Although the rates of preterm birth did not seem to be elevated in initial reports, data suggest pregnant women are at a higher risk of subsequent preterm birth, although this effect is mostly due to clinical intervention based on maternal illness rather than the spontaneous onset of preterm labor ${ }^{4}$.

With respect to the neonate, a national UK surveillance study suggested neonatal SARS-CoV-2 infection is uncommon even in neonates born to mothers with perinatal infection ${ }^{5}$. Similarly, a study from the United States of 116 mothers with confirmed perinatal SARS-CoV-2 infection did not identify any neonatal cases ${ }^{6}$. There are now clear criteria to define mother-to-child transmission of SARS-CoV-2 (https://www.who.int/publications/i/item/WHO2019-nCoV-mother-to-child-transmission-2021.1/), and a small number of individual case reports have documented evidence of vertical transmission ${ }^{7-9}$. The results of these and other studies suggest that vertical transmission is possible, but infrequent.

Although vertical transmission of SARS-CoV-2 is infrequent, the potential immunological perturbations induced in the pregnant mother ${ }^{10}$ might leave an immunological legacy on the newborn with far-reaching consequences. Indeed, evidence exists that perinatal inflammation can modulate the developing immune system of neonates born prematurely ${ }^{11}$. The immune system of the fetus can be altered by the presence of human immunodeficiency virus (HIV) or hepatitis $\mathrm{C}$ virus (HCV) in mothers, either with or without vertical transmission ${ }^{12,13}$; metabolites derived from the maternal intestinal microbiota can shape the immune system of the offspring ${ }^{14}$; and that modulation of the neonatal immune system is associated with diseases in later life ${ }^{15}$. In the few studies assessing the immune status of neonates born to SARS-CoV-2-infected mothers, there has been little evidence of effect in the cellular and humoral immunity of the neonate ${ }^{16}$. Similarly, a small study of SARS-CoV-2 infection during pregnancy has been associated with a cytokine response in the fetal circulation (that is, umbilical cord blood) with no effect on the cellular immune repertoire ${ }^{17}$. However, to our knowledge, none of these studies have included a comprehensive analysis of the cellular immune profile of neonates born to SARS-CoV-2-exposed mothers (at any point in their pregnancy) compared to profiles of neonates born to unexposed mothers. Indeed, it is increasingly challenging to include an appropriate control group in a global pandemic. Additionally, neonatal $\mathrm{T}$ cell responses specific to SARS-CoV-2 peptides are yet to be investigated. Moreover, simultaneous analysis of cytokines and antibody titers in neonates and their paired mothers is lacking.

Here, we assess the immunological status of neonates born to mothers with SARS-CoV-2 who tested positive either during the 2 weeks directly before birth or earlier in pregnancy, compared with those born to mothers who were never exposed to SARS-CoV-2, to identify if there is a legacy of maternal infection and in utero priming of the neonatal immune system.

\section{Results}

Reduced antibody transfer in neonates born to mothers with recent or ongoing infection. Maternal and neonatal characteristics are shown in Table 1, and the group descriptions are available in Fig. 1a. Neonates born to mothers with SARS-CoV-2 exposure (SARS-CoV-2 exposed (SE), $n=30$ ) were grouped into those born

'Peter Gorer Department of Immunobiology, School of Immunology and Microbial Sciences, King's College London, Guy's Hospital, London, UK. 'Department of Women's Health, Guy's and St Thomas' NHS Foundation Trust, St Thomas' Hospital, London, UK. ${ }^{3}$ Department of Infectious Diseases, School of Immunology and Microbial Sciences, King's College London, Guy's Hospital, London, UK. Department of Women and Children's Health, School of Life Course Sciences, Faculty of Life Sciences and Medicine, King's College London, St Thomas' Hospital, London, UK. $₫ e-m a i l:$ deena.gibbons@kcl.ac.uk 


\section{Table 1 | Maternal and neonatal clinical characteristics}

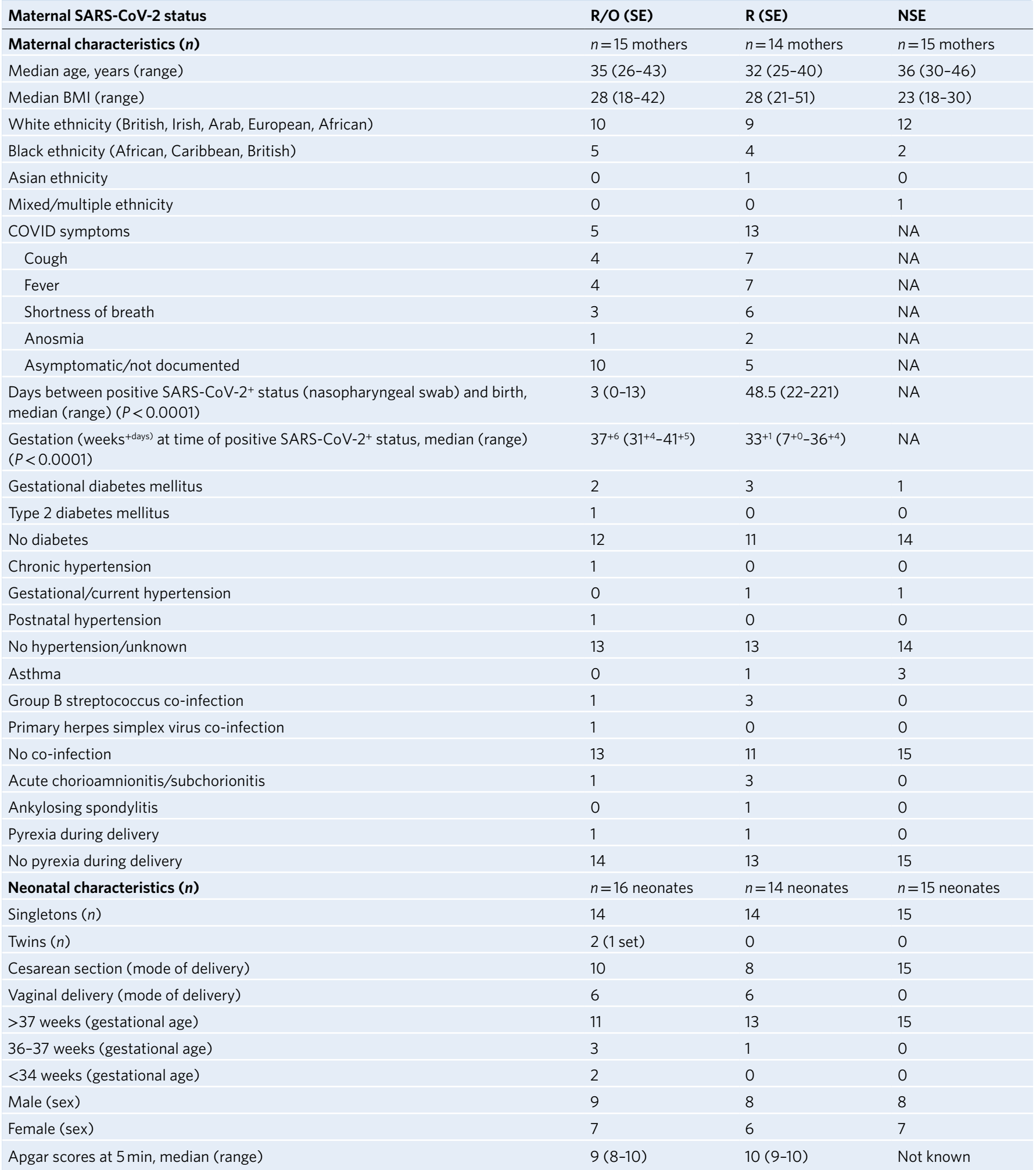

NSE $(n=5)$ samples used in FluoroSpot experiments not included. NA, not applicable.

to mothers with recent or ongoing infection (R/O, identified by positive PCR test within 2 weeks of birth, $n=16$ ), or those born to mothers testing positive earlier in gestation (recovered group $(\mathrm{R})$, determined positive at least 2 weeks before birth, $n=14$, median positive swab $48.5 \mathrm{~d}$ before birth).
In both the recent/ongoing and recovered group, regardless of the time between the positive swab and birth or maternal IgM levels, SARS-CoV-2-specific IgM was not detected in cord plasma, suggestive of a lack of vertical transmission (Fig. 1b). This was true for IgM directed against the spike $(S)$ protein, the receptor-binding domain 


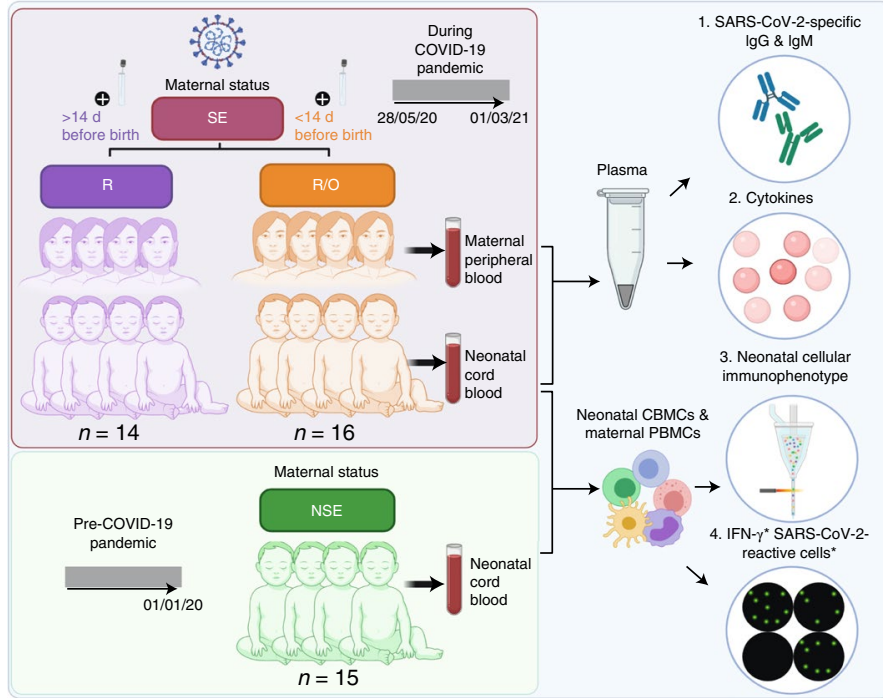

* 8 SE neonates and paired mothers, and 5 additional NSE neonates tested

b

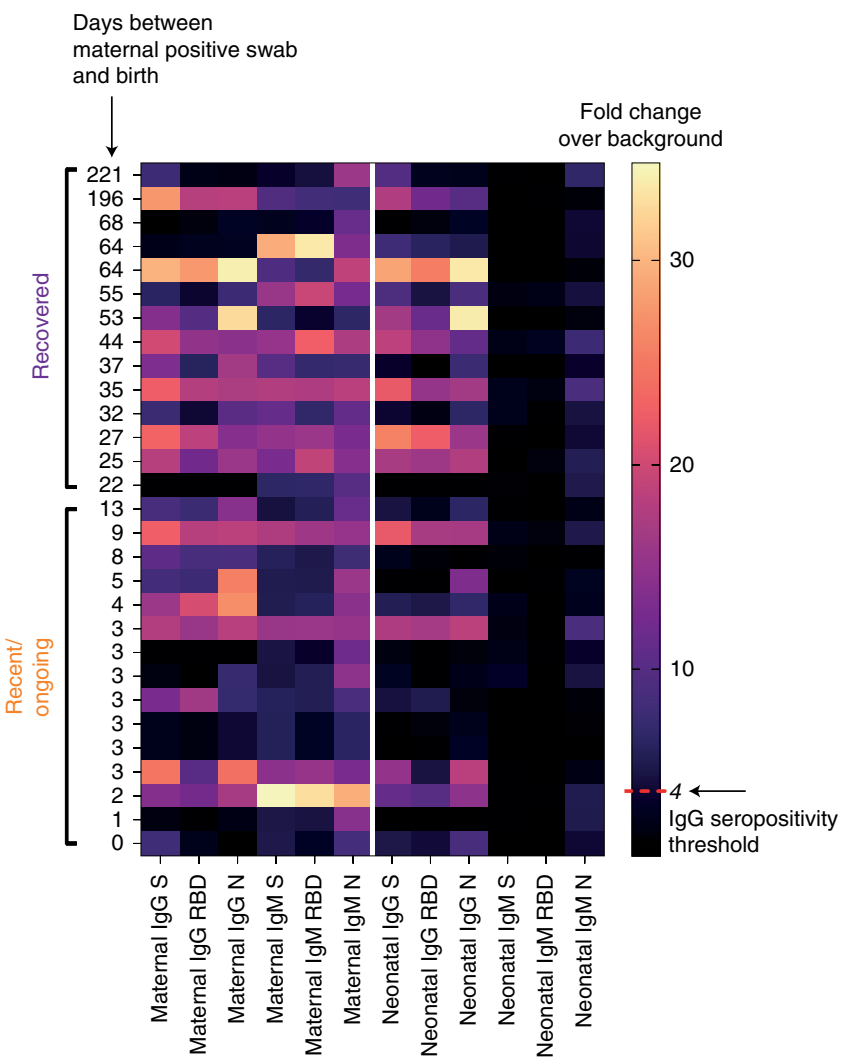

C

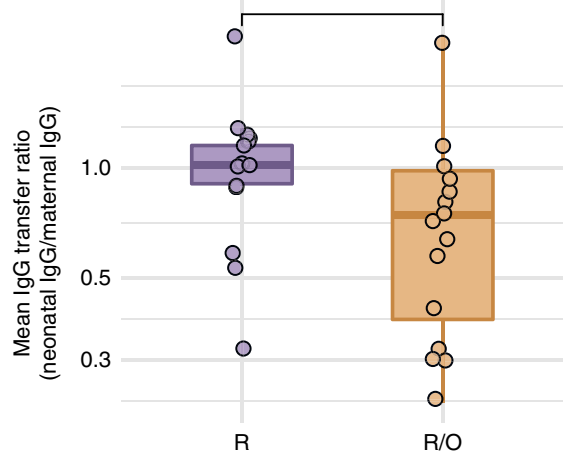

d
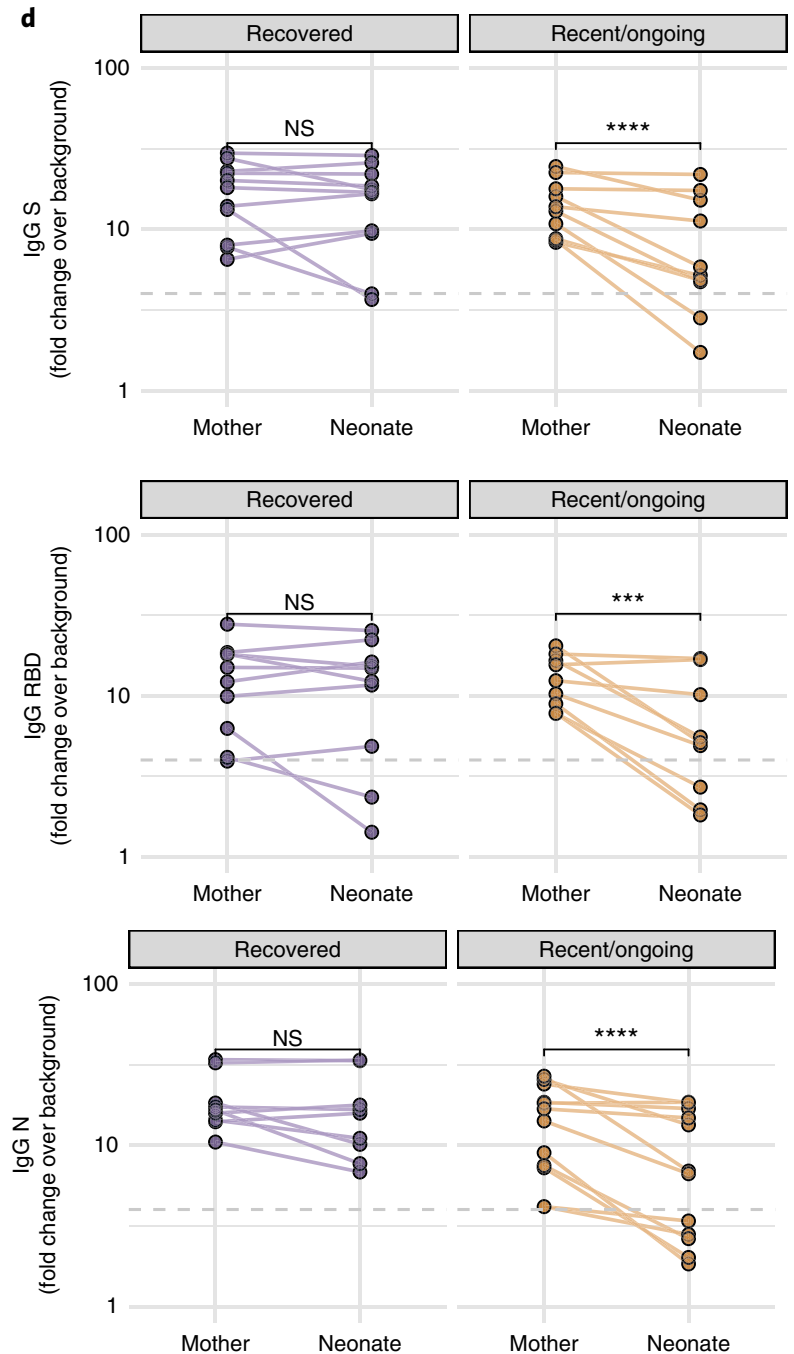

Fig. 1 | Neonates born to SE mothers are IgG ${ }^{+}$IgM- and reduced antibody transfer was observed in the R/O group. a, Study outline illustrating recruitment of neonates and mothers in the SE group $(n=30)$, comprising two groups $(R, n=14 ; R / O, n=16)$, and neonates in the NSE group $(n=15)$. Figure created with BioRender.com. b. Heat map displaying paired maternal and neonatal IgG and IgM levels against S, RBD and N SARS-CoV-2 epitopes measured in plasma from maternal blood and cord blood taken at the time of delivery. Peak lgG/lgM levels (fold change over background) are displayed within the neonate-mother dyads (each row; $\mathrm{R}, n=14 ; \mathrm{R} / \mathrm{O}, n=15$ ), ordered by the number of days between maternal positive nasopharyngeal swab and birth. Fourfold change (over background) was defined as the IgG seropositivity threshold. c, Box plot of the mean IgG (average of IgG S, N and RBD) transfer ratio between the neonates and their paired mothers within the $\mathrm{R}(n=14)$ and R/O $(n=15)$ groups. $\mathbf{d}$, Maternal and neonatal paired peak IgG levels (fold change over background) in dyads with seropositive mothers only (fourfold change over background is indicated by the dashed gray line)

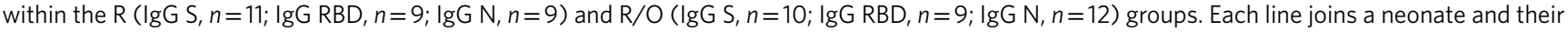
paired mother. Box plots follow standard Tukey representations; the central line denotes the median, upper and lower lines represent the 75th and 25th percentiles, respectively, and whiskers represent 1.5 times the 75 th and 25 th percentiles. $P$ values were assessed by unpaired Wilcoxon rank-sum tests (c) and paired Wilcoxon tests (d). All statistical tests were two-sided and all $P$ values $\left({ }^{\star} P<0.05 ;{ }^{\star \star} P<0.01 ;{ }^{\star \star \star \star} P<0.0001\right)$ were unadjusted. 
(RBD) within the spike protein as well as the nucleoprotein $(\mathrm{N})$ for which background IgM reactivity has been shown to be higher ${ }^{18}$. By contrast, SARS-CoV-2-specific IgG against all three antigens was detected in neonates born to SARS-CoV-2-exposed mothers (Fig. 1b). While there was no significant difference in SARS-CoV2-specific IgG levels in the mother-neonate dyads in the recovered group, there were significantly lower levels of SARS-CoV-2-specific IgG in neonates born to mothers with recent/ongoing infection (Extended Data Fig. 1a). Thus, when the ratio of neonatal immunoglobulin to their paired maternal immunoglobulin (transfer ratio) was calculated for each antigen, the mean transfer ratio of all three antigens was significantly lower in the R/O group (0.79) compared to the R group (1.04; Fig. 1c). This was despite the presence of high levels of maternal IgG in at least some mothers in the $\mathrm{R} / \mathrm{O}$ group (Extended Data Fig. 1a). Indeed, when only comparing IgG levels in neonates born to seropositive mothers, transfer of SARS-CoV2 -specific IgG to the neonate was significantly lower in the $\mathrm{R} / \mathrm{O}$ group (Fig. 1d), whereas again the antibody levels were comparable between mother and neonate in the R group. Antibody transfer did not appear to differ with the sex of the newborn, with both male and female neonates in the $\mathrm{R} / \mathrm{O}$ group having significantly less SARS-CoV-2-specific IgG compared to their seropositive mothers (Extended Data Fig. 1b).

Elevated plasma cytokine levels in mothers with recent or ongoing infection and their neonates. SARS-CoV-2 infection in adults is known to be associated with marked elevation of several plasma cytokines including interferon gamma-induced protein 10 (IP-10), interleukin (IL)-1 $\beta$, CXCL8, IL-6 and IL-10 (refs. ${ }^{19-21}$ ). Plasma cytokine concentrations were assessed both in maternal and cord blood using a multiplex assay. Maternal plasma cytokine analysis revealed significantly elevated IP-10 and IL-1 $\beta$ levels in mothers with recent or ongoing SARS-CoV-2 infection compared to recovered mothers, while IL-10, CXCL8 and IL-6 levels in maternal plasma were similar between the two SE groups (Fig. 2a). When assessing neonatal cytokine levels, IL-10 was significantly elevated in the cord plasma from neonates born to mothers with recent or ongoing infection compared to those born to recovered mothers, where no IL-10 could be detected. CXCL8 levels were also numerically higher in the R/O group (median, $93 \mathrm{pg} \mathrm{ml}^{-1}$ ) compared to the recovered group (median, $28 \mathrm{pg} \mathrm{ml}^{-1}$ ), although this did not reach significance, largely driven by three neonates with undetectable levels (Fig. 2b). However, concentrations of this chemokine were significantly higher in neonates than their paired mothers in both SE groups (Fig. 2c), which was not seen with any of the other cytokines tested. Conversely, neonatal IP-10 was equivalent between mothers and neonates in the recovered group and actually significantly lower in neonates than their paired mothers in the R/O group (Extended Data Fig. 2a). The majority of neonates born to recovered mothers who showed elevated levels of CXCL8 were born by vaginal delivery (Fig. 2d), known to elevate several cytokines ${ }^{22}$. However, there were still notable, albeit insignificant $(P=0.07$, unpaired two-tailed
Kolmogorov-Smirnov test), increases of CXCL8 in neonates born via cesarean section (CS) in the R/O group, compared to the $\mathrm{R}$ group, suggesting this was possibly a neonatal response to maternal SARS-CoV-2 infection.

Spearman rank test identified significant correlations in cytokine levels both within and between maternal and cord blood in both $\mathrm{R}$ and $\mathrm{R} / \mathrm{O}$ groups, although this was much more evident in in the $\mathrm{R} / \mathrm{O}$ group, suggestive of a greater degree of immune co-regulation in recent/ongoing SARS-CoV-2 infection (Fig. 2e). For example, within the R/O group, there were significant correlations between neonatal CXCL8 and IP-10 (Fig. 2f), and maternal IL- $1 \beta$ levels correlated with both neonatal CXCL8 and IP-10 (Fig. 2g,h). Interestingly, in all the SE neonates, maternal IL-1 $\beta$ levels negatively correlated ( $R=-0.49, P=0.016$, two-tailed Spearman correlation test) with days between a positive COVID swab and birth (Fig. 2i), suggesting this cytokine was indicative of recent infection in the mothers. No differences were seen in plasma IL-12p70, granulocyte-macrophage colony-stimulating factor (GM-CSF), interferon (IFN)- $\alpha 2$, IFN- $\lambda 1$, IFN- $\lambda 2 / 3$, IFN- $\beta$, tumor necrosis factor (TNF) and IFN- $\gamma$ in mothers or neonates from either group (Extended Data Fig. 2b).

Recent or ongoing maternal infection affects neonatal immune cells. To investigate the potential impact of maternal SARS-CoV-2 infection on the cellular immune compartment of the neonate at birth, we used multiparametric flow cytometry to phenotype cord blood leukocytes and assess their in vitro functional capacity upon mitogen stimulation (Figs. 3-6). Gating strategies are shown in Extended Data Fig. 3. To establish if maternal SARS-CoV-2 infection altered the developing immune system of the neonate, cellular immune profiles in the combined ( $\mathrm{R}$ and R/O) SARS-CoV2-exposed group (SE; $n=30$ ) were compared to those from term neonates born to healthy mothers collected before the pandemic (non-SARS-CoV-2-exposed (NSE), $n=15$ ) but measured simultaneously. We performed $t$-distributed stochastic neighbor embedding ( $t$-SNE) dimensionality reduction (Fig. 3a) on 91 individual flow cytometry immune parameters and observed that the immune profiles in the SE group clustered away from the immune profiles of the NSE group. Significant correlations (as assessed by two-tailed Spearman correlation tests) between immune parameters appeared different in neonates born to SE mothers compared to NSE mothers (Fig. 3b,c). For example, the significant correlation observed between IFN- $\gamma$-producing natural killer (NK) cells and CD8 ${ }^{+} \mathrm{T}$ cells in the NSE group was completely absent in the SE group, and a significant correlation appeared between the important effector populations, $\mathrm{CD}^{+}$terminal effector memory RA-positive (TEMRA) cells and $\mathrm{CD} 8_{\text {TEMRA }}$ cells in the SE group, where there had been no such correlation in the NSE group (Extended Data Fig. 4a,b). Indeed, when focusing on the immune parameters that drive the biggest differences, we identified that neonates born to mothers in the R/O group separated furthest away from the NSE group, using three-dimensional principal-component analysis (3D PCA; Fig. 4a), and that all three groups tended to segregate based on their maternal

Fig. 2 | Elevated plasma cytokine levels in neonates and paired mothers within the R/O group. a,b, IP-10, IL-1 $\beta$, IL-10, CXCL8 and IL-6 levels in mothers (a) and their neonates (b) within the R group (mothers-all cytokines, $n=13$; neonates-CXCL8 \& IL-6, $n=14, I P-10, n=13 ; I L-1 \beta, n=12 ; I L-10, n=11)$ and the R/O group (mothers-IL-6, $n=15 ;$ IL-10 \& IL-1 $\beta, n=14 ;$ CXCL8 \& IP-10, $n=13$; neonates-IP-10, CXCL8, IL-10 and IL-6, $n=16 ; \mathrm{IL}-1 \beta, n=15$ ) measured in plasma from maternal blood and cord blood taken at the time of delivery. $\mathbf{c}$, Paired maternal and neonatal plasma CXCL8 levels within the R ( $n=13$ ) and the R/O ( $n=14)$ groups. Each line joins a neonate and their paired mother. $\mathbf{d}$, Neonatal CXCL8 levels in babies born via CS (left, $n=18)$ or vaginal delivery (VD; right, $n=12$ ). Horizontal dotted lines represent the minimum (and maximum, for IL-6) detectable concentrations. e, Spearman correlation matrices of all significant $(P<0.05)$ correlations of neonatal and maternal cytokines in the R (left, $n=14)$ and R/O (right, $n=16)$ groups. $\mathbf{f}-\mathbf{i}$, Spearman correlation plots between neonatal IP-10 and CXCL8 (f), maternal IL-1 $\beta$ and neonatal CXCL8 (g), and maternal IL-1 $\beta$ and neonatal IP-10 (h) in the R/O group (f: $n=15, \mathbf{g}$ and $\mathbf{h}: n=14)$ and between maternal IL-1 $\beta$ and days between maternal positive swab in the SE group $(\mathbf{i} ; n=27)$. Plots indicate a generalized linear regression line with shaded $95 \%$ confidence intervals. Associated correlation coefficients and $P$ values are in orange (f-h) and dark red (i). In the cytokine scatterplots ( $\mathbf{a}, \mathbf{b}$ and $\mathbf{d}$ ), the central line represents the median value. $P$ values were assessed by unpaired Kolmogorov-Smirnov tests ( $(\mathbf{a}, \mathbf{b}$ and $\mathbf{d})$ and paired Wilcoxon tests $(\mathbf{c})$. All statistical tests were two-sided and all $P$ values $\left({ }^{\star} P<0.05 ;{ }^{\star \star} P<0.01\right)$ were unadjusted. 
SARS-CoV-2 status upon unbiased hierarchal clustering analysis (Fig. 4b). Moreover, there was no clear clustering based on alternative confounding factors such as sex, ethnicity, mode of delivery and other maternal characteristics such as chorioamnionitis and gestational diabetes (Extended Data Fig. 5). Despite the observation that the neonates did segregate based on their mother's SARS-CoV-2
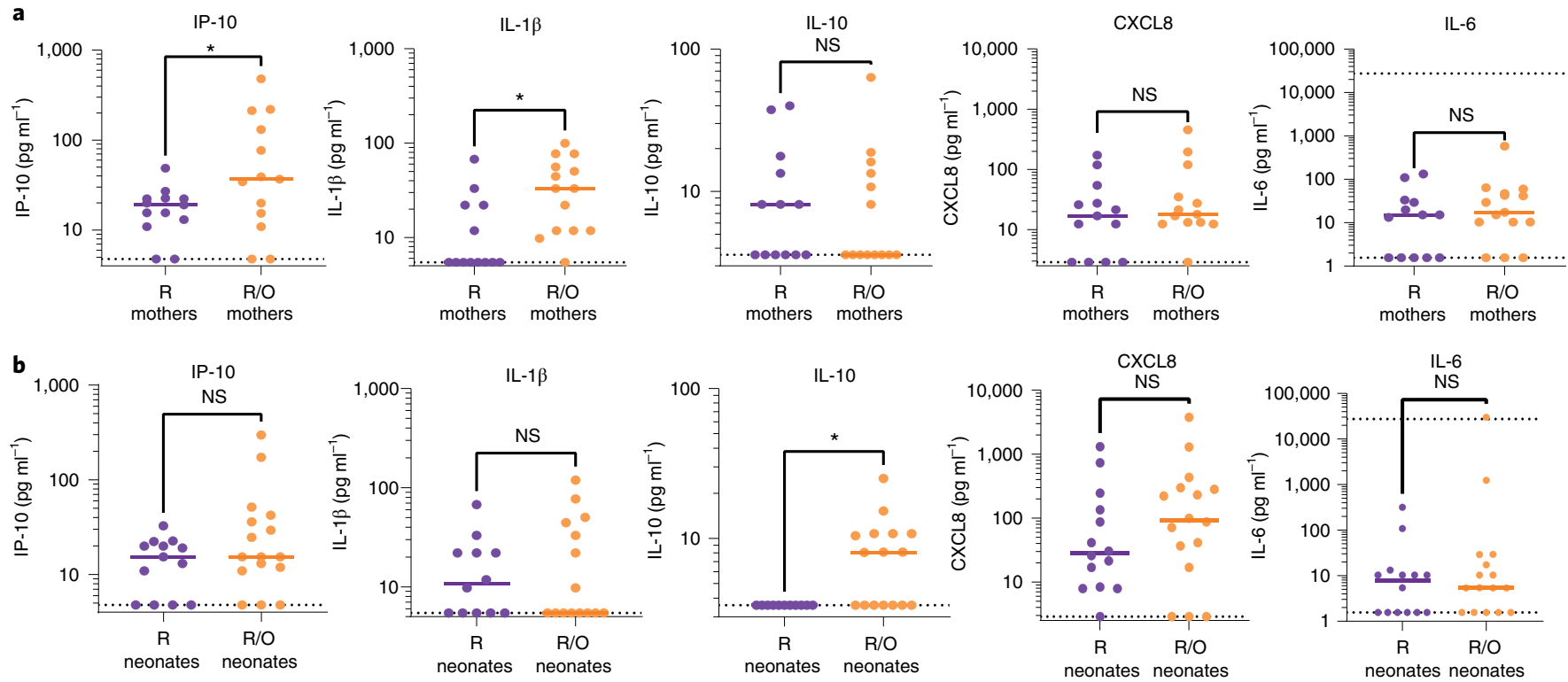

c
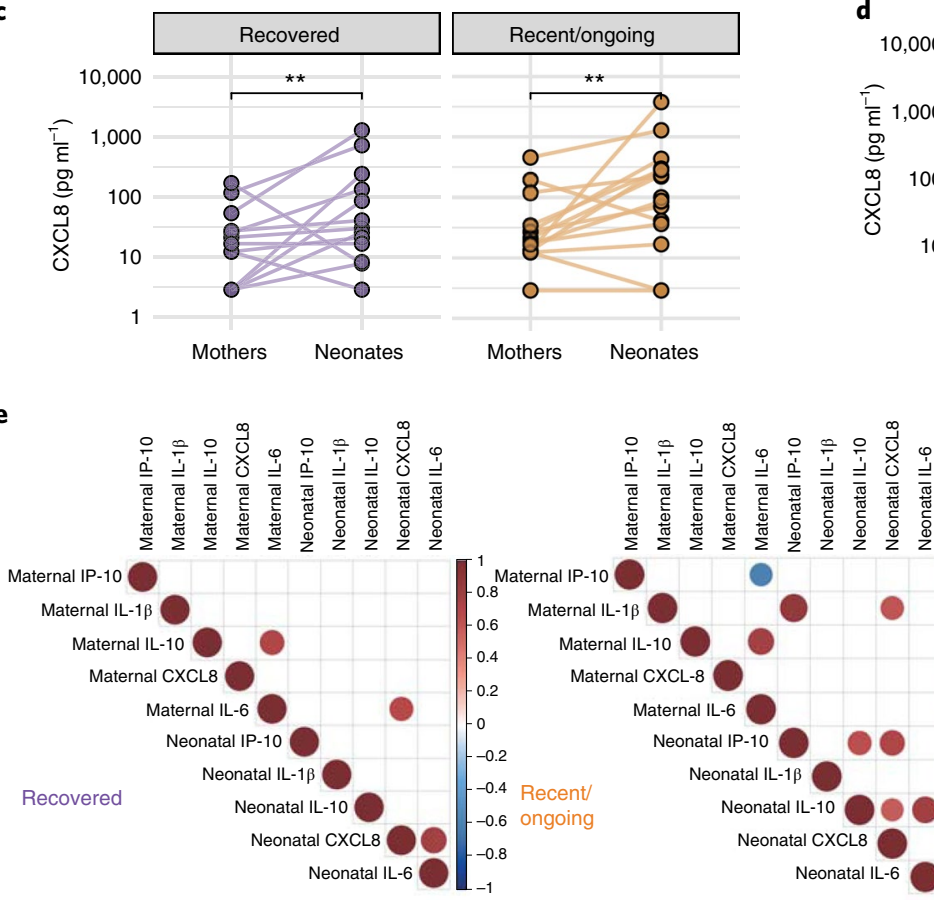

g

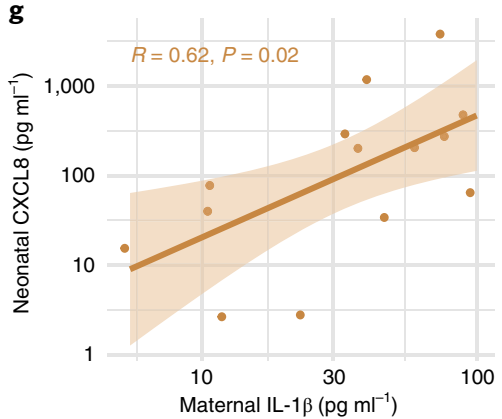

Maternal IL-1 $\beta\left(\mathrm{pg} \mathrm{ml}^{-1}\right)$

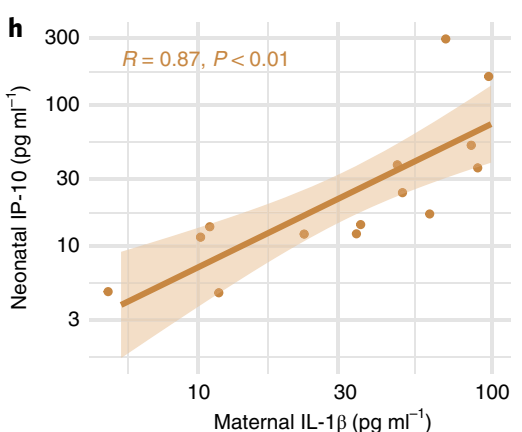

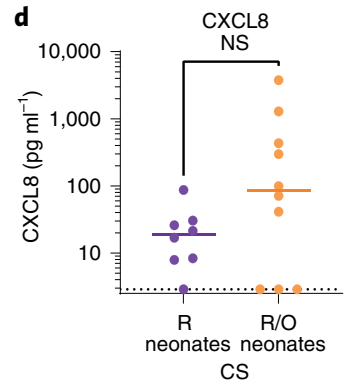

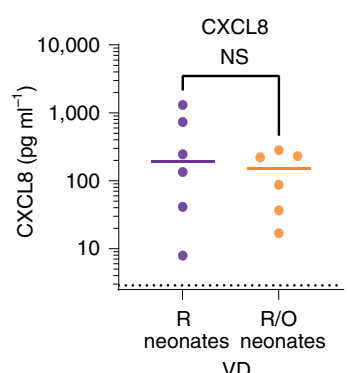

f
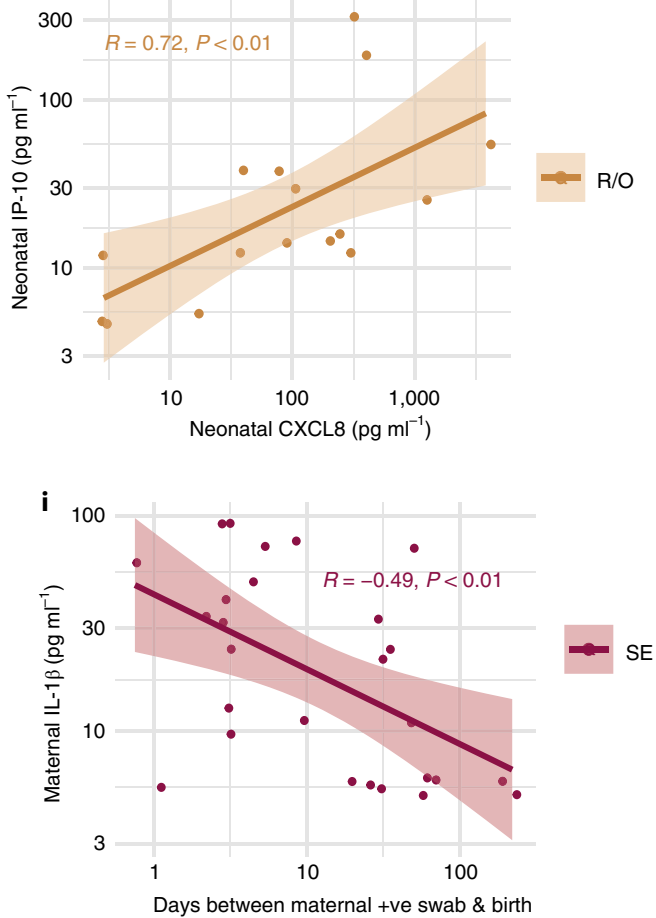


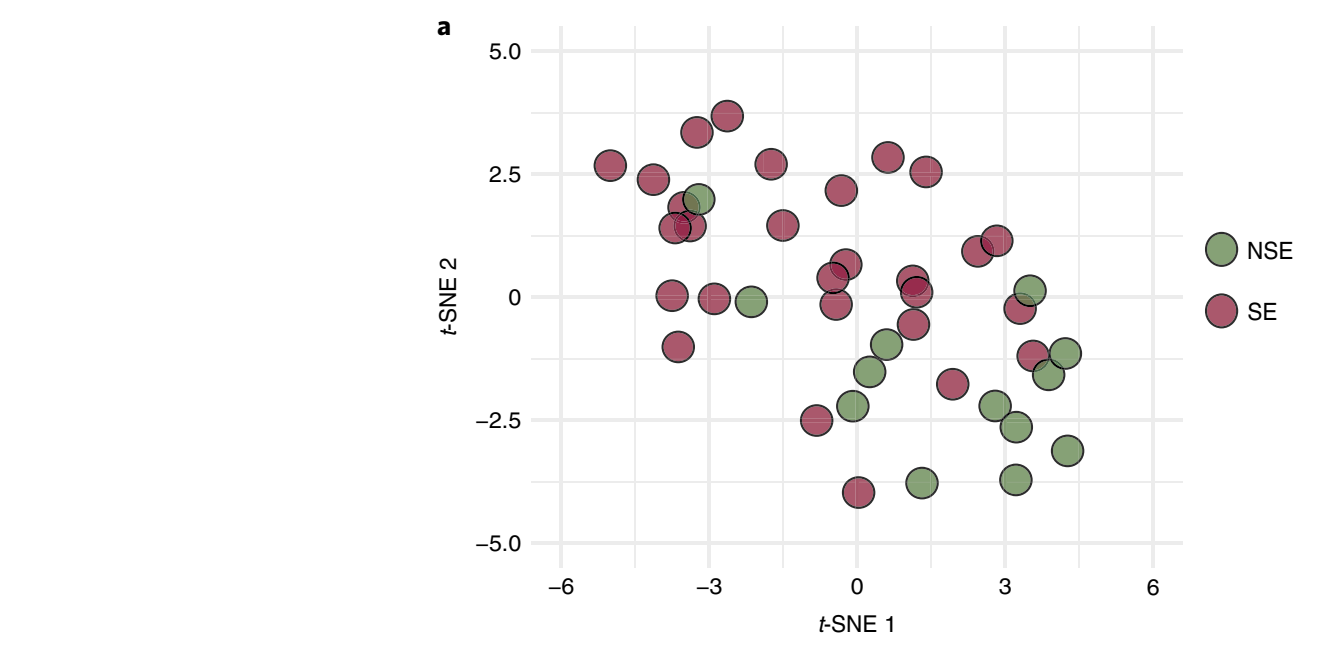

b

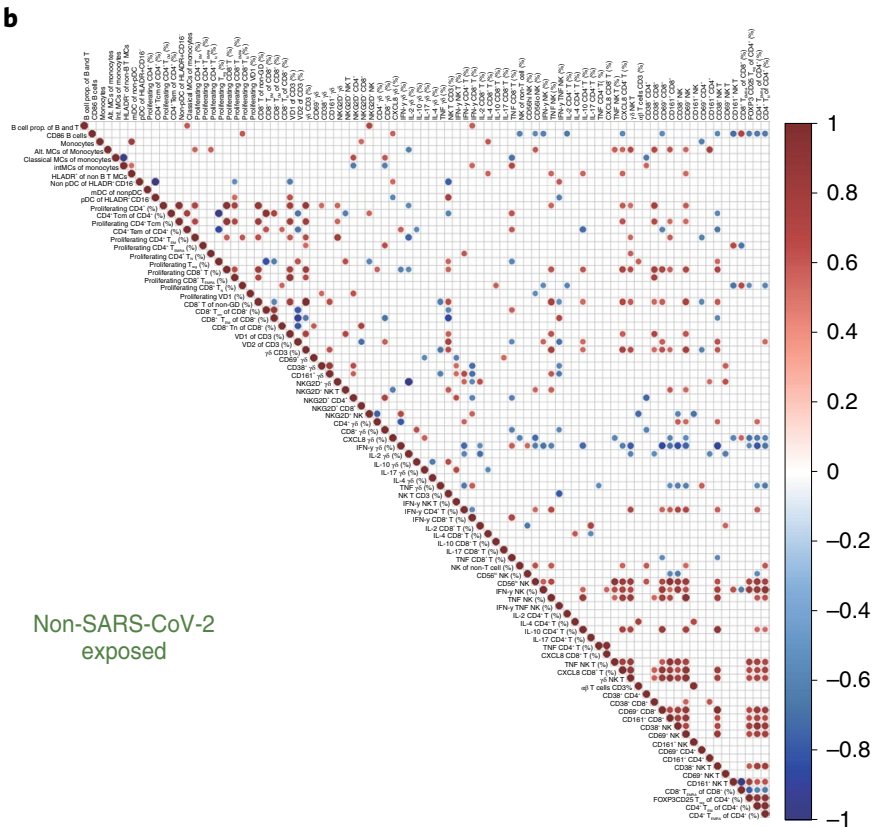

c

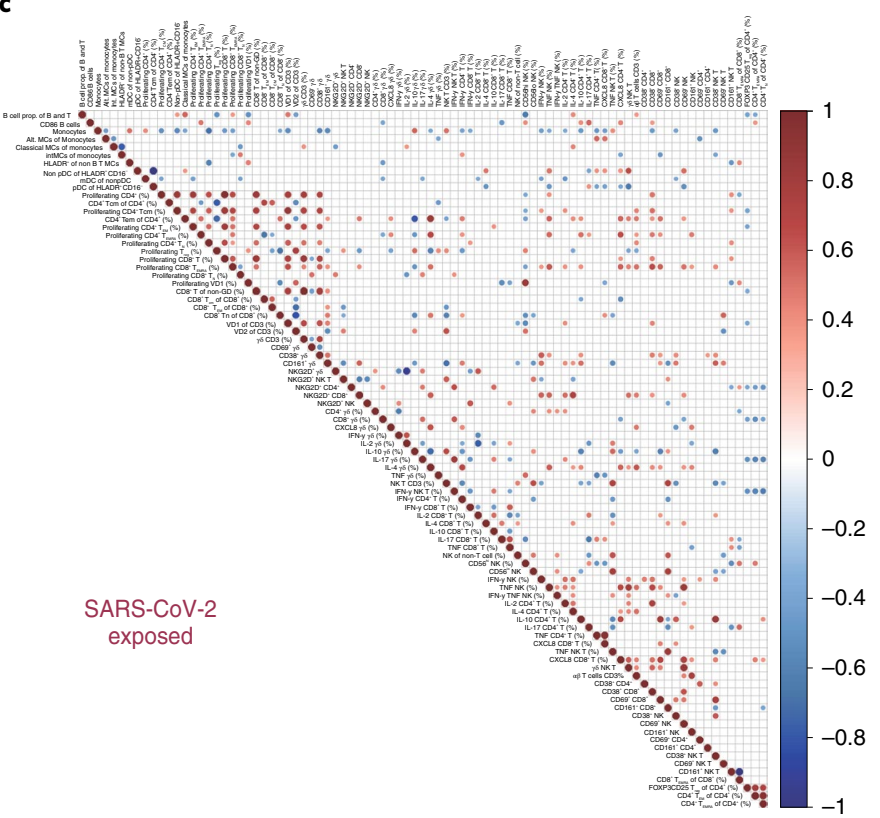

Fig. 3 | Immune differences between neonates born to mothers exposed to SARS-CoV-2 and unexposed mothers. a, $t$-SNE dimensionality reduction of neonatal immune profiles measured from CBMCs in neonates born to SE mothers $(n=28)$ and NSE mothers $(n=13)$. b,c, Spearman correlation matrix of significant $(P<0.05)$ correlations of neonatal cellular populations in the NSE $(n=14 ; \mathbf{b})$ the $\operatorname{SE}(n=30 ; \mathbf{c})$ groups. Spearman correlation tests were two-sided and $P$ values were unadjusted. $T_{E M}$ cell, effector memory $T$ cell; $T_{C M}$ cell, central memory $T$ cell; $T_{N}$ cell, naive $T$ cell. MC, monocyte.

status, many parameters, including those known to be perturbed in the adult response to SARS-CoV-2, were not significantly different between any of the groups. T cell lymphopenia was not observed and, moreover, the relative frequencies of major adaptive lymphocyte subsets in the neonate (for example, $\mathrm{CD} 4^{+}$and $\mathrm{CD}^{+} \alpha \beta$ $\mathrm{T}$ cells, $\gamma \delta$ T cells and B cells) were unaffected by maternal exposure to SARS-CoV-2 and were similar to those seen in the NSE group (Fig. 4c). Whereas these neonatal immune cell populations were not affected in totality by maternal SARS-CoV-2 exposure, more specific changes in adaptive cell populations were still evident. Thus, while the percentage of CD161-expressing $\mathrm{CD}^{+} \mathrm{T}$ cells was equivalent between NSE and R groups, there was a significant increase in this population in neonates born to mothers with recent or ongoing infection (Fig. 4d). Furthermore, the percentage of CD25+FOXP3 ${ }^{+}$ regulatory $\mathrm{T}\left(\mathrm{T}_{\mathrm{reg}}\right)$ cells was significantly increased in neonates from the R/O group compared to the NSE and R groups (Fig. 4e). When assessing all the neonates born to SARS-CoV-2-exposed mothers, the percentage of $\mathrm{T}_{\text {reg }}$ cells significantly correlated with the percentage of $\mathrm{V} \delta 2^{+} \mathrm{T}$ cells (Fig. 4f).

Increased percentages of innate immune cells in neonates born to mothers with recent or ongoing infection. In contrast to the relatively unaffected adaptive immune cell compartment, changes in innate cell subsets were more prominent following maternal SARS-CoV-2 exposure. NK, NK T and innate-like $V \delta 2^{+} \gamma \delta \mathrm{T}$ cells were all significantly elevated in neonates born to mothers with recent or ongoing infection when compared to the NSE group (Fig. $5 \mathrm{a}-\mathrm{c})$. NK and $\mathrm{V} \delta 2^{+} \gamma \delta \mathrm{T}$ cells were also significantly elevated in neonates from the $\mathrm{R} / \mathrm{O}$ compared to $\mathrm{R}$ group. There was also a change in monocyte populations with significantly enhanced percentages of alternative monocytes and subsequently significantly reduced $\mathrm{CD}_{3} 8^{+}$classical monocytes in neonates born to mothers with recent or ongoing infection compared to the NSE group (Extended Data Fig. 6a). These cells were, for the most part, not 
significantly elevated in neonates born to mothers with previous SARS-CoV-2 exposure (R) compared to the NSE counterparts, consistent with reactive neonatal responses to recent or ongoing SARS-CoV-2 infection. Indeed, when looking at all the neonates born to SE mothers, raised percentages of NK cells showed a negative correlation $(R=-0.39, P=0.04$, two-tailed Spearman correlation test) with days from positive SARS-CoV-2 swab result to birth, further suggesting this was a neonatal response to maternal infection (Extended Data Fig. 6b). Interestingly, within the SE group, there was a significant correlation between the levels of cord blood CXCL8 and the percentage of NK T cells (Fig. 5d) and the concentrations of cord blood CXCL8 and NK cell activation (as assessed by CD69 expression; Fig. 5e) suggesting these are key immune markers associated with the neonatal response to maternal infection.

Increased cytokine functionality in neonates born to mothers exposed to SARS-CoV-2. When assessing functionality by intracellular cytokine staining (ICS) after polyclonal activation, the ability of immune cells to produce cytokines upon stimulation was significantly elevated in neonates born to SE mothers. Consistent with changes observed in plasma cytokine concentrations and cellular immune composition, enhanced cytokine production by neonatal immune cells was associated with maternal SARS-CoV-2 infection. Indeed, cytokine potential was significantly enhanced in several different cell types in neonates born to mothers previously exposed to SARS-CoV-2 (at any time point). Thus, the percentages of $\mathrm{CD}^{+}$, $\mathrm{CD}^{+}, \gamma \delta, \mathrm{NK}$ and $\mathrm{NK} \mathrm{T}$ cells producing TNF were significantly increased in neonates in the R/O group compared to the NSE group, but also significantly increased in all these cell types in the $\mathrm{R}$ group compared to the NSE counterparts (Fig. 6a). The percentage of cells expressing IFN- $\gamma$ was also significantly increased in $\mathrm{CD}^{+}, \mathrm{CD}^{+}, \gamma \delta$ and NK T cells from the R/O group compared to the NSE group (Fig. $6 \mathrm{~b}$ ), and significant increased percentages of IFN- $\gamma$-producing $\mathrm{CD}^{+}$ and NK T cells were seen in the R group compared to the NSE group (Fig. 6b). IL-17-expressing cells were also increased albeit to a lesser extent, with significant increases in $\mathrm{CD} 4^{+}$cells expressing IL-17 in the R/O group compared to the NSE group, and the percentages of IL-17-producing $\gamma \delta$ T cells were significantly increased in R/O and R groups compared to the NSE group (Extended Data Fig. 6c). The percentages of $\mathrm{CD}^{+}, \mathrm{CD}^{+}$or $\gamma \delta$ T cells making CXCL8 was equivalent in all the groups (Extended Data Fig. 6d). The observed enhanced cytokine functionality (TNF and IFN- $\gamma$ ) in $\mathrm{CD}^{+}{ }^{+} \mathrm{T}$ cells from neonates born to mothers exposed to SARS-CoV-2 significantly correlated with effector memory $\mathrm{CD}^{+}{ }^{+} \mathrm{T}$ cells (Fig. 6c,d). Indeed, CD38 expression, known to decrease during maturation ${ }^{11}$, negatively correlated with both TNF and IFN- $\gamma$-producing $\mathrm{CD} 4^{+}$and $\mathrm{CD} 8^{+}$cells in the SE group $(R=-0.45$ to $-0.59, P \leq 0.01$, two-tailed Spearman correlation tests; Extended Data Fig. 6e). Owing to limited numbers of mothers with severe disease, we were unable to establish if the extent of immune imprinting was related to maternal infection status.

Viral peptide responses detected in mothers exposed to SARSCoV-2 but rarely in neonates. To assess whether the increased cytokine functionality observed in cells from SE neonates was due to the presence of SARS-CoV-2-specific T cells, we stimulated cord or peripheral blood mononuclear cells (PBMCs) from neonates and their seropositive mothers $(n=8)$, respectively, with SARS-CoV2 -specific peptides and assessed responses via IFN- $\gamma$ FluoroSpot assay. All, except one, of the SARS-CoV-2-exposed mothers had positive responses against all the SARS-CoV-2 peptide pools with a combined median to the matrix and nucleocapsid, spike 1 ( $\mathrm{N}$ terminus) and spike 2 (C terminus) peptide pools of $36 \mathrm{IFN}-\gamma^{+}$spots per $10^{6}$ cells (range, $\left.15-100\right)$ in R/O mothers $(n=4)$ and a combined median to the three peptide pools of 15 IFN- $\gamma^{+}$spots per $10^{6}$ cells (range, $1-70)$ in $\mathrm{R}$ mothers $(n=4)$. However, no responses were detected to any SARS-CoV-2 peptides in the R/O, R or NSE $(n=5)$ neonatal groups with the exception of one neonate in the $\mathrm{R} / \mathrm{O}$ group where a weak IFN- $\gamma$ response to SARS-CoV-2 spike ( $\mathrm{N}$ terminus) and matrix/nucleocapsid peptides was observed (Fig. 7).

\section{Discussion}

Our study investigated the immune profiles of neonates born to mothers with SARS-CoV-2 exposure. While we did not observe SARS-CoV-2-specific IgM in the neonates and hence, by definition, saw limited vertical transmission of SARS-CoV-2, we did find multiple immunological perturbations within the neonate associated with maternal SARS-CoV-2 exposure during pregnancy, many of which were associated with recent or ongoing infections. Taken together, our findings are suggestive of an immunological legacy imprinted on the neonate following maternal SARS-CoV-2 exposure, with potential far-reaching consequences.

Although we did not directly assess neonates for the presence of SARS-CoV-2, we did assess SARS-CoV-2-specific IgM levels, which were negligible in all 30 neonates born to SARS-CoV-2-exposed mothers, suggestive of no vertical transmission. SARS-CoV2 -specific IgG was, however, transferred to the neonates from their mothers, suggestive of the transfer of protective immunity. There was a correlation between maternal and neonatal SARS-CoV-2 IgG levels in mother-neonate dyads in both groups as previously suggested $^{23}$. However, even though for many pathogens, umbilical cord titers of IgG at normal-term delivery are higher than those in maternal blood ${ }^{24,25}$, there were reduced levels of SARS-CoV2 -specific IgG in neonates born to mothers with recent or ongoing infection compared to their paired mothers. This did not appear to be a threshold issue, as many mothers exhibited high levels of SARS-CoV-2-specific IgG, which was not transferred efficiently to their newborn. Reduced placental transfer of SARS-CoV-2-specific immunoglobulin has been observed previously ${ }^{26}$, possibly due to altered glycosylation ${ }^{27}$. Furthermore, reduced maternal SARS-CoV2 -specific antibody titers and impaired placental antibody transfer were also noted in pregnancies with a male fetus ${ }^{28}$, although there did not appear to be any sex bias in our dataset. It is currently unclear whether antibodies induced via vaccination, as opposed to natural infection, differ in terms of their glycosylation status and subsequent placental transfer, which is an important area of research. Vaccination to SARS-CoV-2 in the second and third trimester did elicit placental transfer of antibodies, with a reduced transfer ratio observed in the last trimester ${ }^{29}$. Our study adds further evidence

Fig. 4 | Recent or ongoing maternal SARS-CoV-2 infection affects neonatal immune cells. a, 3D PCA dimensionality reduction of neonatal immune profiles in the R/O $(n=15), \mathrm{R}(n=13)$ and NSE $(n=13)$ neonatal groups. PC1, PC2 and PC3 explain $20.6 \%, 11.8 \%$ and $8.4 \%$ of the variance, respectively. b. Dendrogram cluster heat map of 45 flow cytometry immune populations in neonates within the NSE ( $n=14), \mathrm{R}(n=14)$ and R/O ( $n=15)$ groups. $\mathbf{c}$, Box plots displaying the proportions of $\alpha \beta$ T cells, CD4 ${ }^{+}$T cells, CD8 ${ }^{+}$T cells and $\gamma \delta$ T cells (NSE, $\left.n=15 ; R, n=14 ; R / O, n=16\right)$, and the proportion of $B$ cells (NSE, $n=14 ; \mathrm{R}, n=14 ; \mathrm{R} / \mathrm{O}, n=16)$. d,e, Box plots displaying the proportions of CD161+CD8+ ${ }^{+}$cells and FOXP3+CD25+ $\mathrm{T}_{\text {reg }}$ cells $(\mathrm{NSE}, n=15 ; \mathrm{R}, n=14$; $\mathrm{R} / \mathrm{O}, n=16)$. $\mathbf{f}$, Spearman correlation plot in the SE group $(n=30)$ of neonatal proportions of FOXP3 ${ }^{+} \mathrm{CD} 25^{+} \mathrm{T}_{\text {reg }}$ cells and $\mathrm{V} \delta 2^{+} \gamma \delta \mathrm{T}$ cells. Data indicate a generalized linear regression line with shaded $95 \%$ confidence intervals. Associated correlation coefficients and $P$ values are in dark red. All box plots follow standard Tukey representations; the central line denotes the median, upper and lower lines represent the 75th and 25th percentiles, respectively, and whiskers represent 1.5 times the 75 th and 25 th percentiles. $P$ values were assessed by unpaired Wilcoxon rank-sum tests (c-e). All statistical tests were two-sided and all $P$ values $\left({ }^{\star} P<0.05\right)$ were unadjusted. 
suggesting that the second trimester may represent more opportune vaccination timing, at least with respect to the transfer of passive immunity to the neonate.
In utero exposure to environmental factors, infection and/ or maternal inflammation is increasingly recognized to affect the developing immune system and subsequent responses both to infec- a

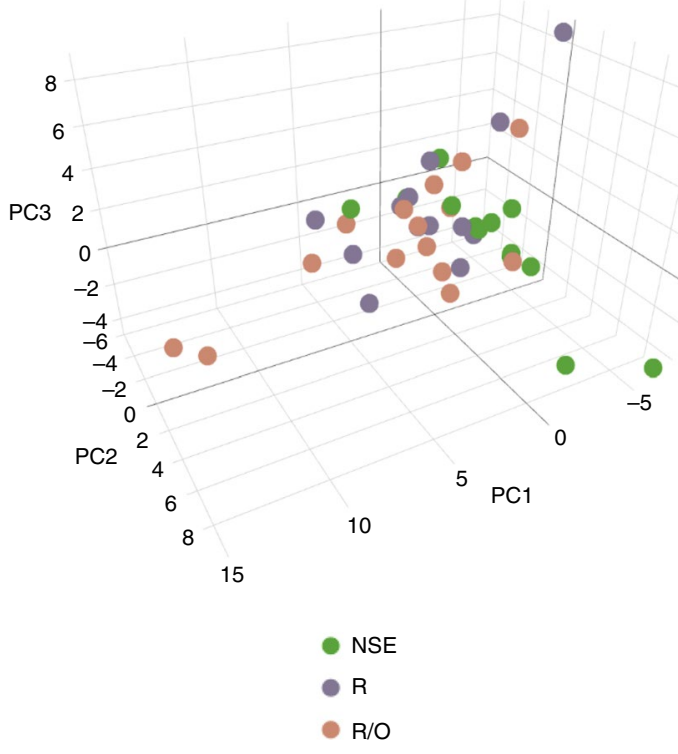

c
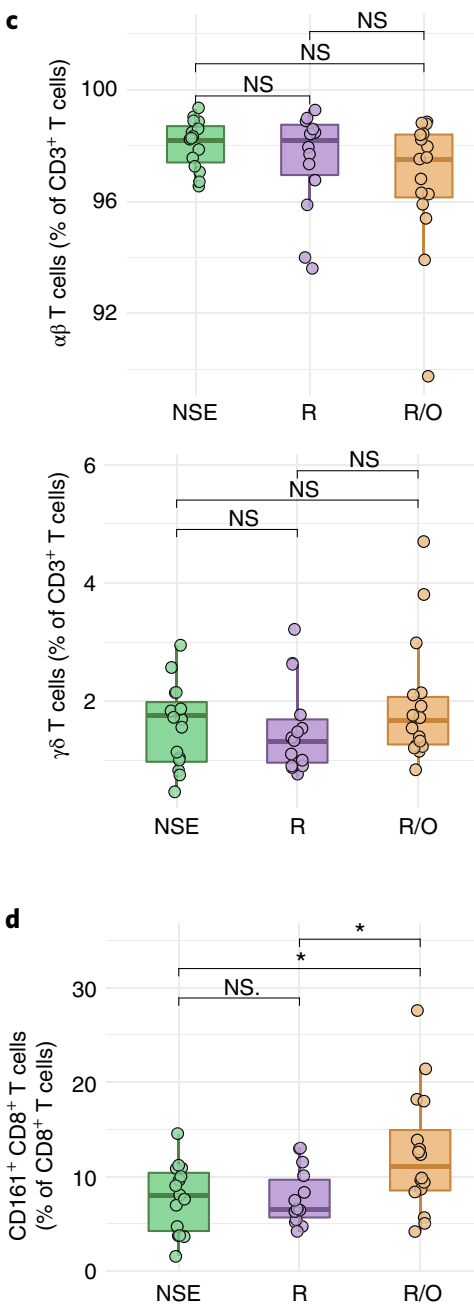

b
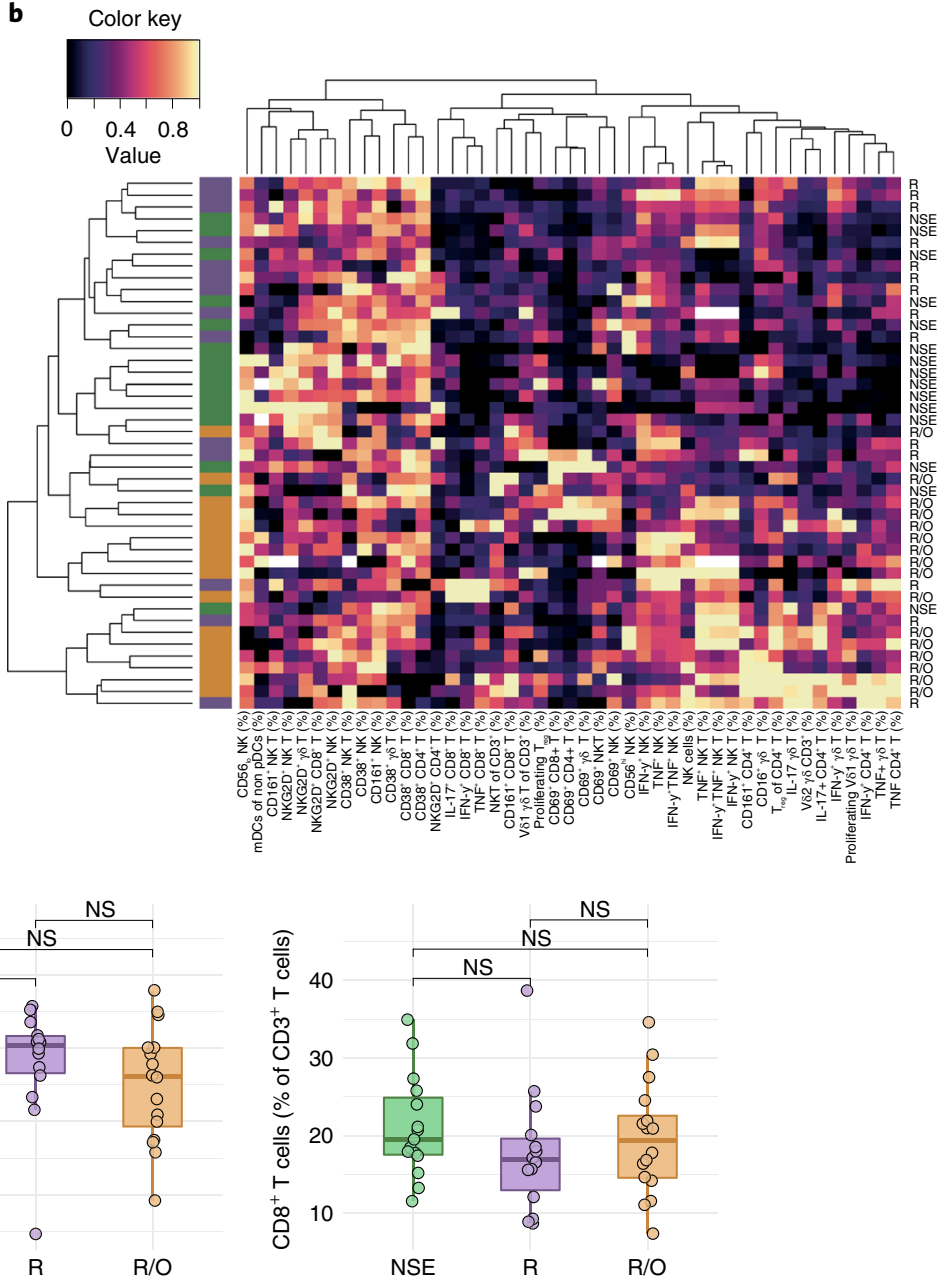

Non-SARS-CoV-2 exposed

Recovered

Recent/ongoing

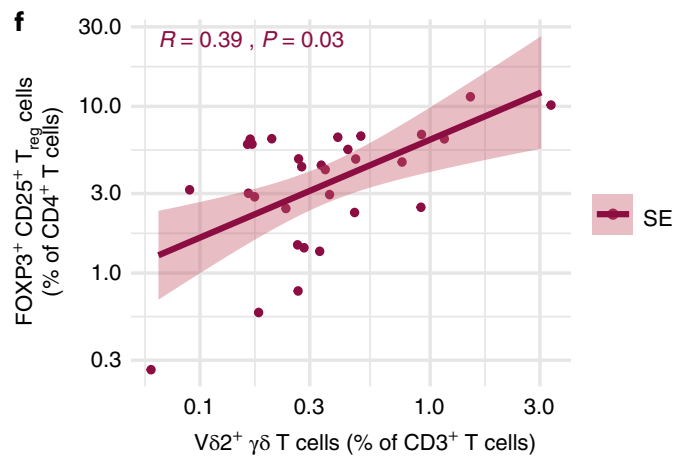


a
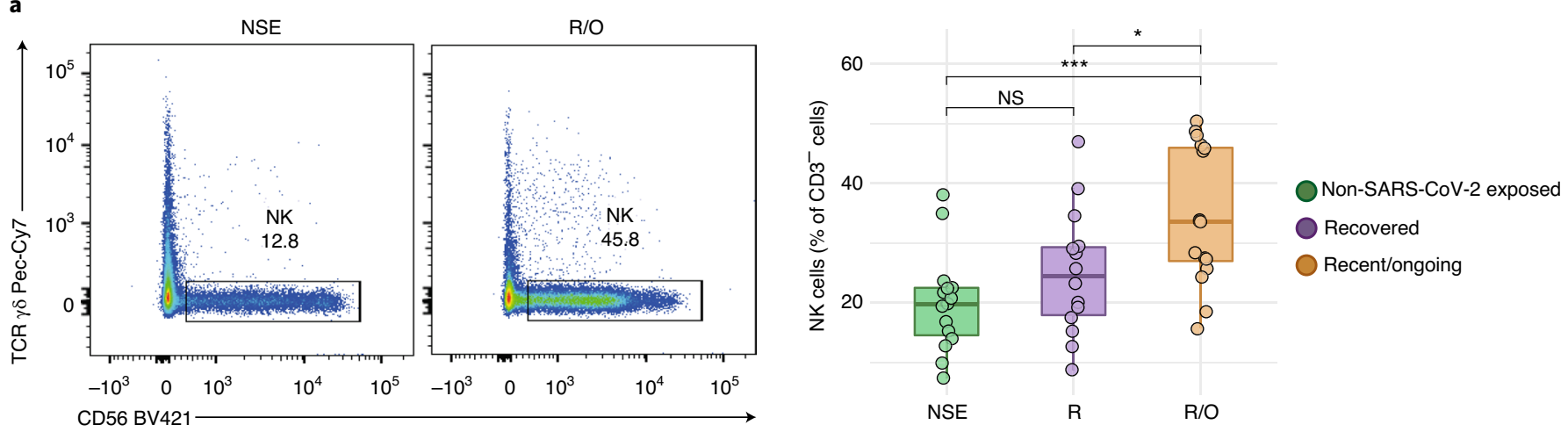

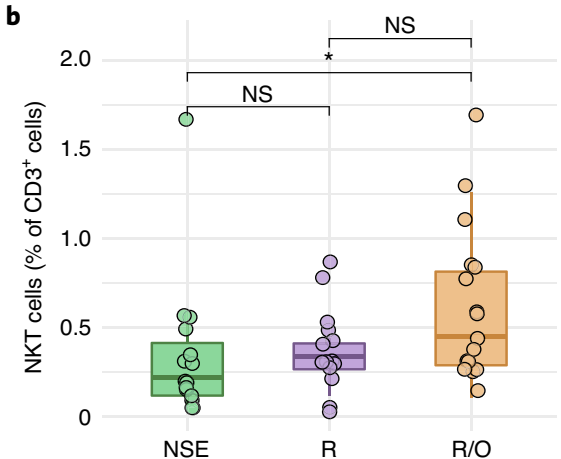

e

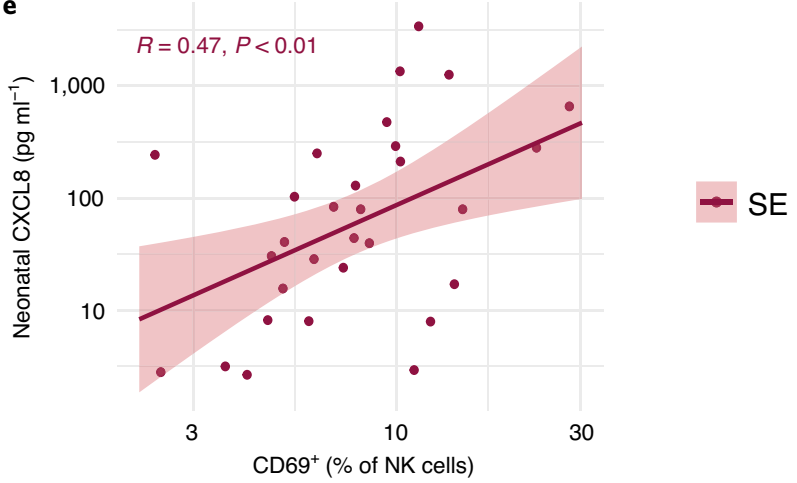

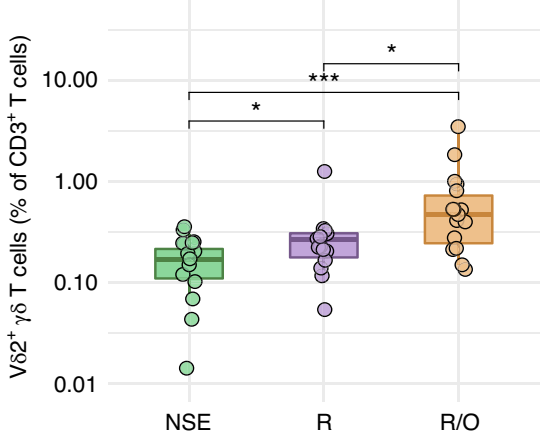

SE

Fig. 5 | Increased proportions of innate immune cells in neonates born to mothers with recent or ongoing infection. a, Representative flow cytometry plots of the percentage of CD56+ NK cells (of CD3- live cells) in an NSE and R/O neonate, measured from CBMCs, and the box plots for the total neonates (NSE, $n=15 ; \mathrm{R}, n=14 ; \mathrm{R} / \mathrm{O}, n=16$ ). b,c, Box plots displaying the proportions of NK T cells and V $\delta 2^{+} \gamma \delta \mathrm{T}$ cells $(\mathrm{NSE}, n=15 ; \mathrm{R}, n=14 ; \mathrm{R} / \mathrm{O}, n=16)$. d,e, Spearman correlation plots in the SE group $(n=30)$ between neonatal CXCL8 and the proportions of NK T cells (d) or CD69+ NK cells (e). Plots indicate a generalized linear regression line with shaded $95 \%$ confidence intervals. Associated correlation coefficients and $P$ values are in dark red. All box plots follow standard Tukey representations; the central line denotes the median, upper and lower lines represent the 75 th and 25 th percentiles, respectively, and whiskers represent 1.5 times the 75 th and 25 th percentiles. $P$ values were assessed by unpaired Wilcoxon rank-sum tests (a-c). All statistical tests were two-sided and all $P$ values $\left({ }^{\star} P<0.05 ;{ }^{\star \star \star} P<0.001\right)$ were unadjusted.

tion $^{30}$, immune-mediated diseases ${ }^{31}$ and neurodevelopmental problems ${ }^{32}$. Indeed, long-term effects cannot be ruled out as observed in survivors after in utero exposure to the 1918 (Spanish) influenza pandemic ${ }^{33}$. Perhaps unsurprisingly, cord plasma of neonates born to mothers with recent or ongoing infection, expressed elevated concentrations of some cytokines known to be associated with inflammation and COVID-19, consistent with placental immune activation $^{19}$. Elevated levels of IP-10, IL-6, IL-10, CXCL8 and IL-1 $\beta$ have been associated with adult infection ${ }^{20,21}$, with increased IL- 6 and IL-10 also associated with severity in SARS-CoV-2 infection in children ${ }^{34}$. Interestingly, this conventional COVID-19 signature was skewed more toward IL-10 (and to a lesser extent, CXCL8) in the neonates born to mothers with recent or ongoing infection. Indeed, increased IL-10 was observed in the R/O group, suggesting a potential imbalance between anti-inflammatory and pro-inflammatory cytokines. Theoretically, the increased concentrations of cytokines in the neonate could be explained by transfer of maternal cytokines through the placental tissues. Indeed, neonates with raised IP-10 concentrations were born to mothers who had high plasma IP-10 measurements, although in general concentrations were significantly lower in neonates compared to mothers. However, in the case of cord plasma CXCL8, a chemokine that correlated strongly with maternal IL-1 $\beta$ (previously associated with maternal SARS-CoV-2 infection $^{35}$ ), levels were significantly higher than those observed in 
a
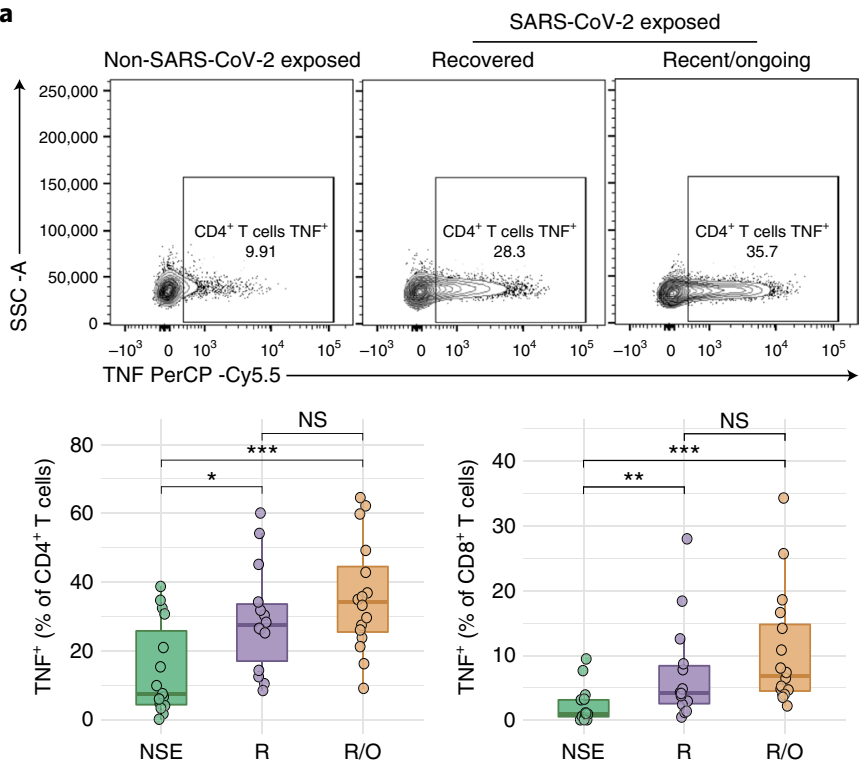

b
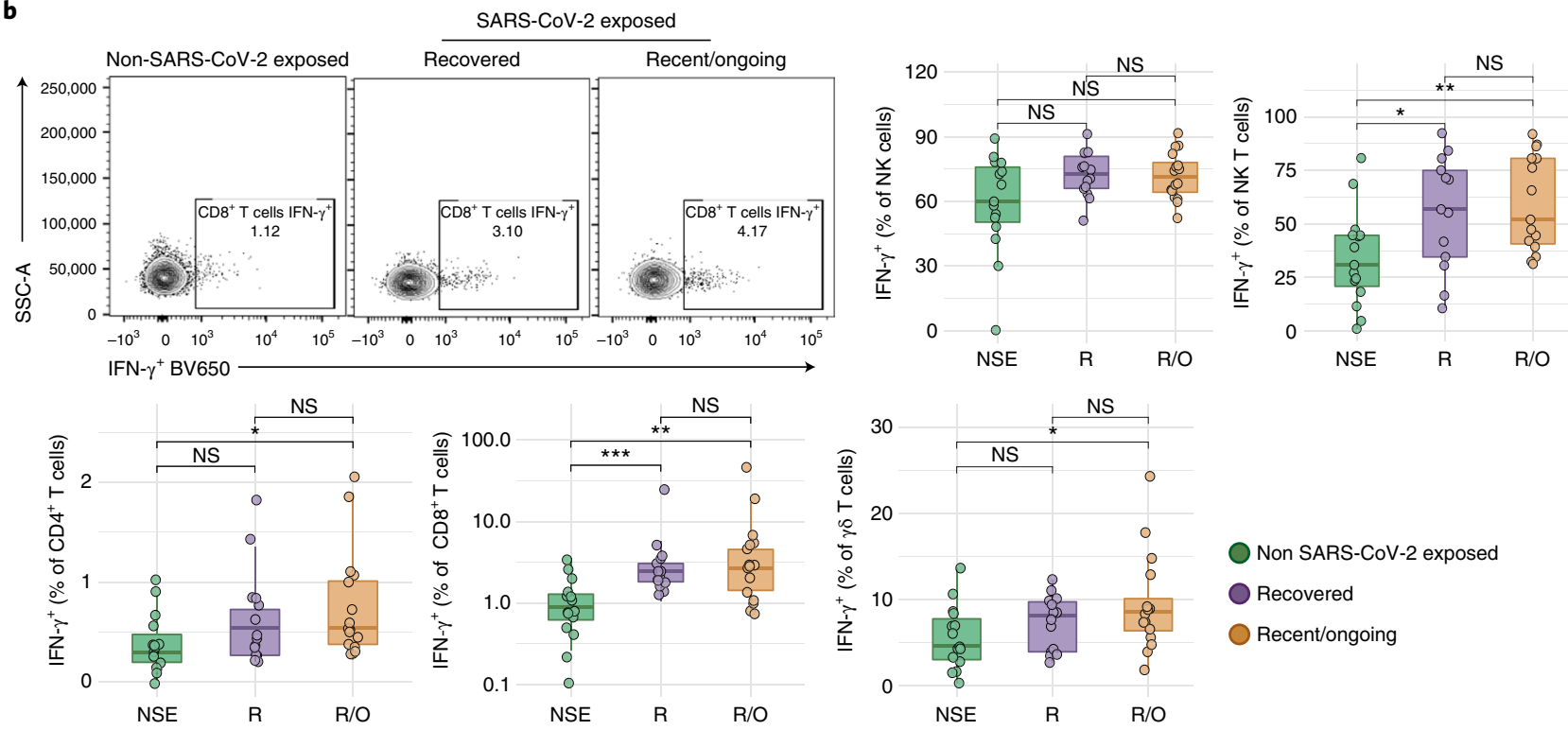

Recovered

Recent/ongoing

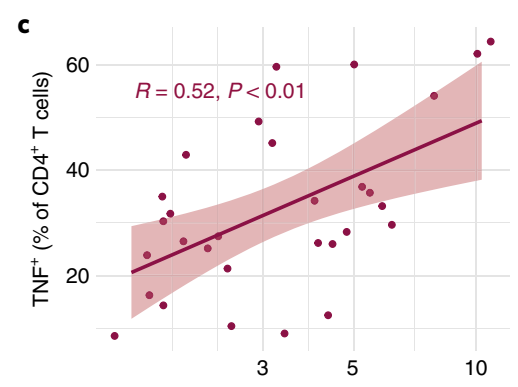

$\mathrm{CD} 4 \mathrm{~T}_{\mathrm{EM}}\left(\%\right.$ of $\mathrm{CD} 4^{+} \mathrm{T}$ cells $)$

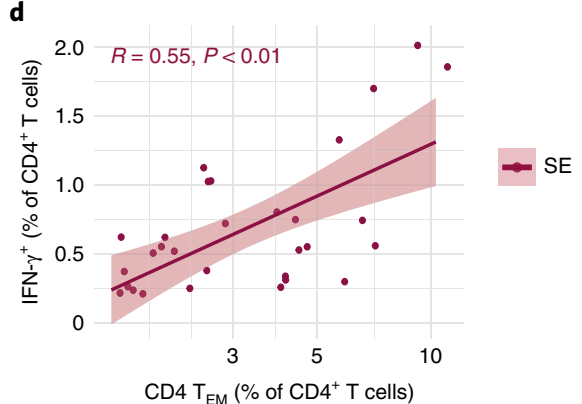

Fig. 6 | Increased percentages of cytokine-producing cells in neonates born to mothers exposed to SARS-CoV-2 after polyclonal stimulation.

$\mathbf{a}, \mathbf{b}$, Representative flow cytometry plots of the percentage of TNF+ CD4 ${ }^{+} \mathrm{T}$ cells $(\mathbf{a})$ and the percentage of IFN- $\gamma^{+} \mathrm{CD} 8^{+} \mathrm{T}$ cells $(\mathbf{b})$ in an NSE, R and R/O neonate, measured from CBMCs. Box plots displaying the proportions of $\mathrm{TNF}^{+}(\mathbf{a})$ and IFN- $\gamma^{+}(\mathbf{b}) \mathrm{CD} 4^{+} \mathrm{T}$ cells, CD $8^{+} \mathrm{T}$ cells, $\gamma \delta \mathrm{T}$ cells and NK cells (NSE, $n=15 ; R, n=14 ; R / O, n=16$ ), as well as NK T cells (NSE, $n=15 ; R, n=13 ; R / O, n=15$ ), following polyclonal stimulation (phorbol 12-myristate 13-acetate (PMA; $\left.10 \mathrm{ng} \mathrm{ml}^{-1}\right)$, ionomycin $\left(1 \mu \mathrm{g} \mathrm{ml} l^{-1}\right)$, brefeldin $\mathrm{A}\left(20 \mathrm{ng} \mathrm{ml}^{-1}\right)$ and monensin solution $(2 \mu \mathrm{M})$ at $37^{\circ} \mathrm{C}$ for $\left.4 \mathrm{~h}\right)$. c, d, Spearman correlation plots between neonatal $C D 4^{+} T_{E M}$ cells $/ \mathrm{TNF}^{+} \mathrm{CD} 4^{+} \mathrm{T}$ cells $(\mathbf{c}), \mathrm{CD} 4^{+} \mathrm{T}_{\mathrm{EM}}$ cells/IFN- $\gamma^{+} \mathrm{CD} 4^{+} \mathrm{T}$ cells $(\mathbf{d})$ in the SE group $(n=30)$. Plots indicate a generalized linear regression line with shaded $95 \%$ confidence intervals. Associated correlation coefficients and $P$ values are in dark red. All box plots follow standard Tukey representations; the central line represents the median, the upper and lower lines represent the 75th and 25th percentiles, respectively, and whiskers represent 1.5 times the 75 th and 25 th percentiles. $P$ values were assessed by unpaired Wilcoxon rank-sum tests (a and $\mathbf{b}$ ). All tests were two-sided and all $P$ values $\left({ }^{\star} P<0.05 ;{ }^{\star \star} P<0.01 ;{ }^{\star \star \star} P<0.001\right)$ were unadjusted. 

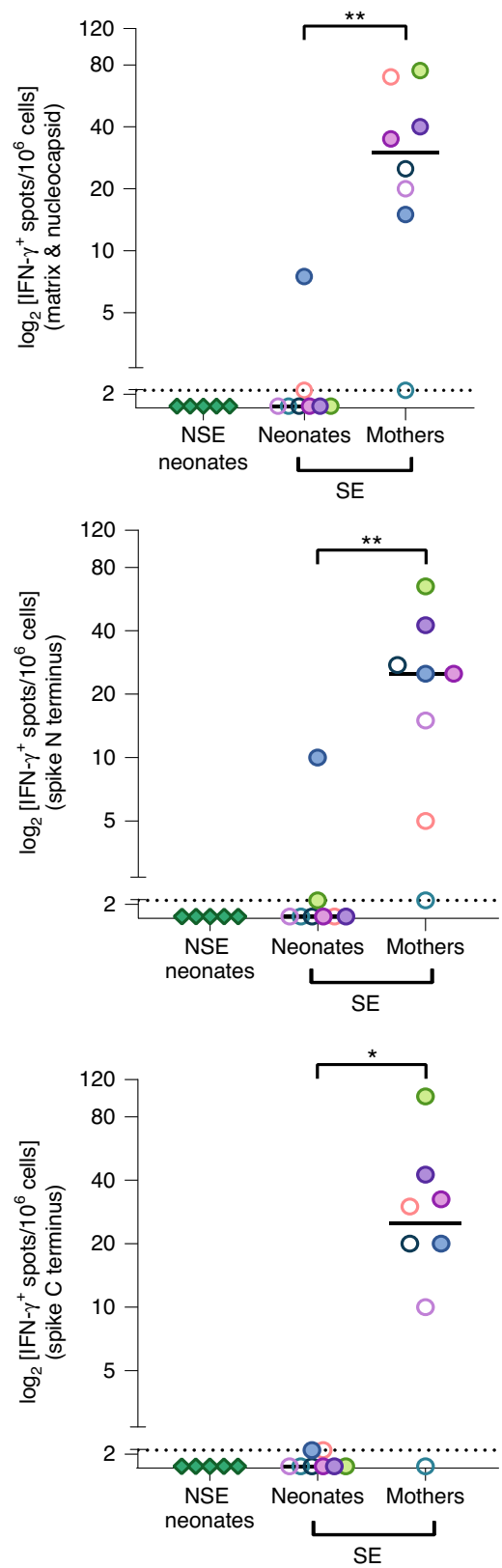

b IFN- $\gamma$ status

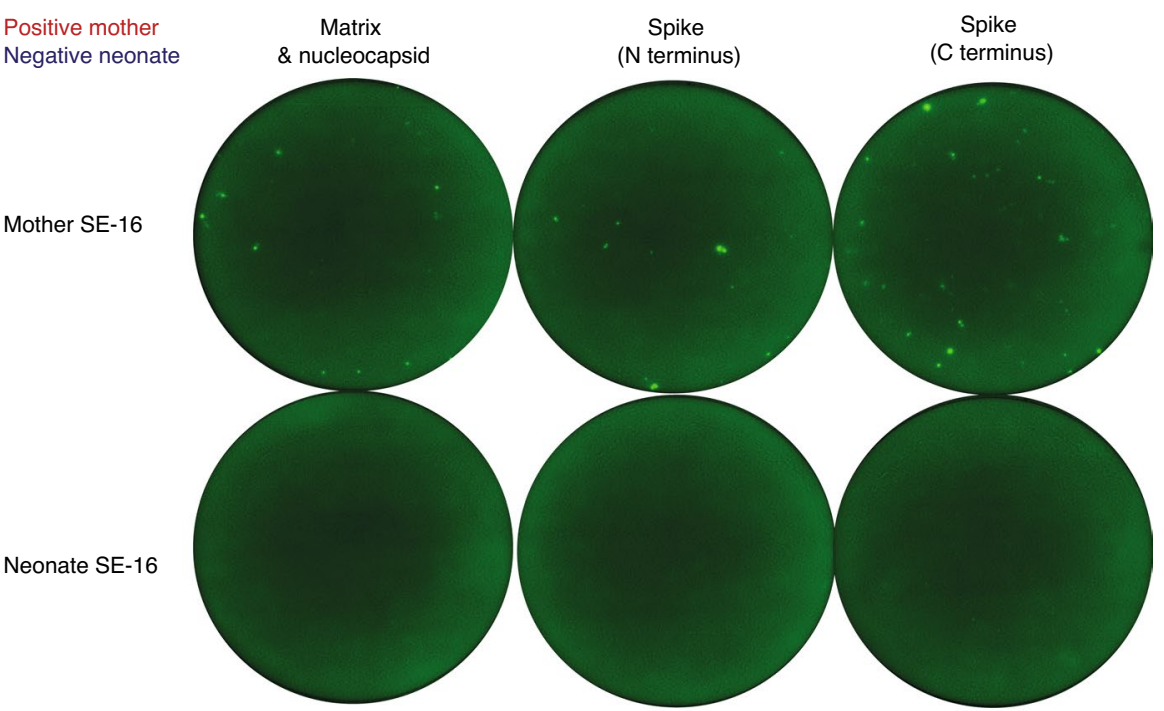

IFN- $\gamma$ status

Positive mother Positive neonate
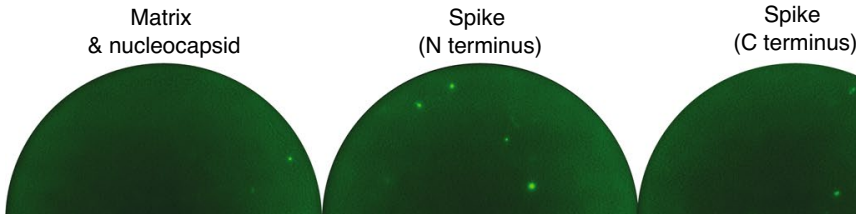

Mother SE-07
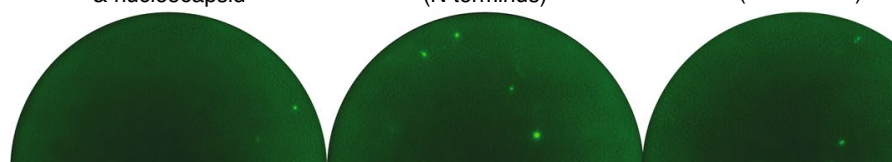

Neonate SE-07
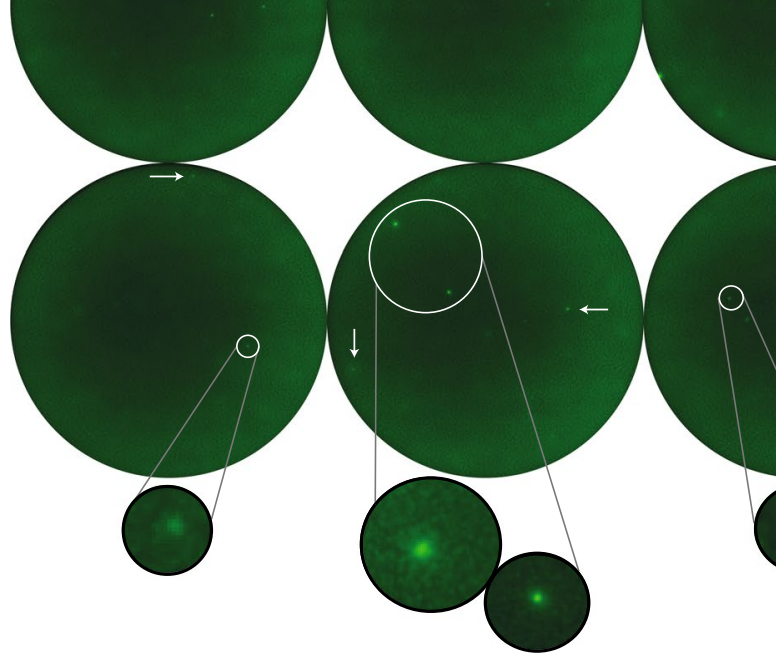

\begin{tabular}{|c|c|c|c|c|c|}
\hline$\diamond$ NSE-16 & & & & - SE-07 & \\
\hline $\begin{array}{l}\text { - NSE-17 } \\
\text { NSE-18 }\end{array}$ & NSE & SE-05 & $\mathrm{R}$ & - SE-08 & $B / 0$ \\
\hline - NSE-19 & & O SE-21 & & O SE-04 & $\pi / 4$ \\
\hline NSE-20 & & O SE-22 & & SE-16 & \\
\hline
\end{tabular}

Fig. 7 | SARS-CoV-2-specific IFN- $\boldsymbol{\gamma}$ responses in mothers and neonates. a, IFN- $\boldsymbol{\gamma}^{+}$spots per $10^{6}$ cells detected by FluoroSpot assay following mononuclear cell stimulation with matrix and nucleocapsid, $\mathrm{N}$ terminus spike and $\mathrm{C}$ terminus spike peptide pools for $24 \mathrm{~h}$ at $37^{\circ} \mathrm{C}$ in $\mathrm{NSE}$ neonates ( $n=5$; diamonds), SE mothers and their paired neonates ( $n=8$ pairs) grouped based on the maternal SARS-CoV-2 status (R/O, filled circles; $\mathrm{R}$, open circles). Individual colors are paired to show the mother-neonate dyads. b. Representative FluoroSpot images of IFN- $\gamma$ (FITC channel) in a mother with positive responses and their paired neonate with negative responses (top) and in the mother-neonate dyad where both individuals had positive responses. White arrows indicate additional IFN- $\gamma^{+}$spots in the neonate that have not been enlarged. Horizontal dotted lines represent the threshold level for positivity. In the scatterplots, the central line represents the median value. $P$ values were assessed by paired Wilcoxon tests between paired SE mothers and their neonates (a). All statistical tests were two-sided and all $P$ values $\left({ }^{\star} P<0.05 ;{ }^{\star \star} P<0.01\right)$ were unadjusted.

their mothers, suggesting that at least some of these elevated cytokines were neonatally derived, and a direct response to maternal infection. Elevated cord plasma cytokines, including CXCL8, have been previously observed in some neonates born to SARS-CoV2-positive mothers ${ }^{17}$. Similarly, increased cord plasma CXCL8 has been observed after in utero exposure to infection ${ }^{36,37}$, suggesting that maternal infection can directly influence fetal CXCL8. Raised neonatal inflammatory markers do appear to be related to the maternal infection status at the time of birth, as such profiles are less obvious in neonates born to mothers with recovered infection. 
Indeed, where we did see slight elevations in CXCL8 in the recovered group, this could be explained by the mode of delivery, as labor is known to drive elevation of these cytokines ${ }^{22}$.

In contrast to COVID-19 infection in adults where reductions in peripheral $\mathrm{NK}, \mathrm{NK} \mathrm{T}, \mathrm{V} \delta 2^{+}$and mucosal-associated invariant $\mathrm{T}$ cells have been observed ${ }^{20,38,39}$, neonates born to mothers exposed to SARS-CoV-2 actually exhibited elevated percentages of NK, NK $\mathrm{T}$ and $\mathrm{V} \delta 2^{+} \gamma \delta \mathrm{T}$ cells and also CD161-expressing CD8 ${ }^{+} \mathrm{T}$ cells (the majority of which are likely to be mucosal-associated invariant $\mathrm{T}$ cells). These innate-like cells may be responding to the inflammatory cytokine milieu in the context of maternal infection potentially as a protective response in the neonate vis-à-vis their likely beneficial role in severe adult COVID-19 (ref. ${ }^{38}$ ). Immune activation of these cells has been seen at the maternal-fetal interface ${ }^{19}$, and although a previous report suggested there was no elevation of NK cells in neonates born to mothers with COVID-19 (ref. ${ }^{16}$ ), this was only compared to reported reference levels and there was no direct comparator group in their study.

As well as alterations in these cell populations, we also identified enhanced cytokine potential upon in vitro stimulation. This was observed not only in neonates born to mothers with recent or ongoing infection but also in those born to recovered mothers, which suggests potential in utero priming of the immune response. Whether the immune profiles identified would be similar in neonates directly infected with SARS-CoV-2 after birth is unclear and this could not be assessed in our study. The percentages of $\mathrm{CD} 4^{+}, \mathrm{CD} 8^{+}, \mathrm{NK}, \mathrm{NK}$ $\mathrm{T}$ or $\gamma \delta \mathrm{T}$ cells that produced TNF (or IFN- $\gamma$ and IL-17 to a lesser extent) were significantly greater in neonates born to mothers who were exposed to SARS-CoV-2. At birth, neonatal T cells predominantly produce CXCL8 with a limited capacity for IFN- $\gamma$ and IL-17 production, which increases with age ${ }^{40}$. Thus, these findings may reflect some accelerated maturation of the neonatal immune system induced in utero by maternal SARS-CoV-2 infection. Much of this enhanced cytokine functionality correlated with other markers of immune maturation such as increased percentages of memory $\mathrm{T}$ cells and decreased $\mathrm{T}$ cell expression of CD38. IFN- $\gamma$ expression is controlled by epigenetic mechanisms in neonates ${ }^{41}$, so it is tempting to speculate that maternal SARS-CoV-2 infection may have induced some epigenetic changes in these loci. Indeed, maternal exposure to polycyclic aromatic hydrocarbons directly altered this locus in cord blood mononuclear cells (CBMCs) ${ }^{42}$, and other data suggest that epigenetic modifications during gestation can shape the future development of diseases like obesity, type 2 diabetes, allergy, asthma and infections ${ }^{43}$. Increased proportions of cytokine-producing $\mathrm{T}$ cells have also been observed following in utero exposure to infection ${ }^{44,45}$. Certain antigens are known to cross the placenta, and fetal priming of the immune system has been observed in response to bacteria detected in fetal organs ${ }^{46}$. Our observation that there was no specific reactivity to SARS-CoV-2 peptides in the majority of SE neonates tested (despite maternal positivity) suggests that the enhanced cytokine responsiveness detected is due to indirect exposure to inflammation/cytokines priming the immune response during fetal development. Nevertheless, SARS-CoV-2-reactive cells were detected in one neonate. This may corroborate that vertical transmission, albeit infrequent, can occur. Indeed, viremia is associated with severity ${ }^{47}$, and this neonate expressed the highest plasma levels of CXCL8, IL- 6 and IL-10 of any of the neonates and was born to a pyrexic mother with placental abruption, which may have aided any viral transmission. By the same token, potential reactivity to viruses without obvious direct transmission has also been suggested by reduction in $\mathrm{T}$ cell receptor diversity observed in HIV-exposed uninfected neonates ${ }^{12}$

Taken together, these data strongly suggest that maternal SARS-CoV-2 infection shapes the immune profile of neonates to different extents, depending on the time of exposure, and that this may be associated both with and without vertical transmission of SARS-CoV-2. We identified a transient response to maternal inflammation in the form of increased cytokines in cord plasma but also altered immune cell functionality in neonates exposed to SARS-CoV-2 at any point during gestation, suggesting some immune imprinting. While the etiology of the observed immune perturbations in the neonate remains unclear, the consequences could be far reaching. A hyper-inflammatory response similar to multisystem inflammatory syndrome in children has been observed in a neonate following in utero exposure to SARS-CoV-2 (mother infected 9 weeks before birth) with no evidence of direct neonatal infection ${ }^{48}$.

Long-term follow-up of the newborns in our study would establish if maternal exposure to SARS-CoV-2 has a long-lasting impact on the child. These data may also have implications regarding the vaccination regimen for pregnant women. Indeed, the reduced transfer of protective antibodies to the neonate we observed in those born to mothers with recent infection may suggest second trimester or early third trimester vaccination is preferable.

\section{Online content}

Any methods, additional references, Nature Research reporting summaries, source data, extended data, supplementary information, acknowledgements, peer review information; details of author contributions and competing interests; and statements of data and code availability are available at https://doi.org/10.1038/ s41590-021-01049-2.

Received: 27 May 2021; Accepted: 14 September 2021;

Published online: 6 October 2021

\section{References}

1. Sutton, D., Fuchs, K., D’Alton, M. \& Goffman, D. Universal screening for SARS-CoV-2 in women admitted for delivery. N. Engl. J. Med. 382, 2163-2164 (2020)

2. Allotey, J. et al. Clinical manifestations, risk factors, and maternal and perinatal outcomes of coronavirus disease 2019 in pregnancy: living systematic review and meta-analysis. BMJ 370, m3320 (2020).

3. Kadiwar, S. et al. Were pregnant women more affected by COVID-19 in the second wave of the pandemic? Lancet 397, 1539-1540 (2021).

4. Martinez-Perez, O. et al. The association between SARS-CoV-2 infection and preterm delivery: a prospective study with a multivariable analysis. $B M C$ Pregnancy Childbirth 21, 273 (2021).

5. Gale, C. et al. Characteristics and outcomes of neonatal SARS-CoV-2 infection in the UK: a prospective national cohort study using active surveillance. Lancet Child Adolesc. Health 5, 113-121 (2021).

6. Salvatore, C. M. et al. Neonatal management and outcomes during the COVID-19 pandemic: an observation cohort study. Lancet Child Adolesc. Health 4, 721-727 (2020).

7. Vivanti, A. J. et al. Transplacental transmission of SARS-CoV-2 infection. Nat. Commun. 11, 3572 (2020).

8. Fenizia, C. et al. Analysis of SARS-CoV-2 vertical transmission during pregnancy. Nat. Commun. 11, 5128 (2020).

9. Dong, L. et al. Possible vertical transmission of SARS-CoV-2 from an infected mother to her newborn. JAMA 323, 1846-1848 (2020).

10. Chen, G. et al. Immune response to COVID-19 during pregnancy. Front. Immunol. 12, 675476 (2021).

11. Kamdar, S. et al. Perinatal inflammation influences but does not arrest rapid immune development in preterm babies. Nat. Commun. 11, 1284 (2020).

12. Gabriel, B. et al. Analysis of the TCR repertoire in HIV-exposed but uninfected infants. Sci. Rep. 9, 11954 (2019).

13. Babik, J. M., Cohan, D., Monto, A., Hartigan-O’Connor, D. J. \& McCune, J. $M$. The human fetal immune response to hepatitis $C$ virus exposure in utero. J. Infect. Dis. 203, 196-206 (2011).

14. Gomez de Aguero, M. et al. The maternal microbiota drives early postnatal innate immune development. Science 351, 1296-1302 (2016).

15. Torow, N. \& Hornef, M. W. The neonatal window of opportunity: setting the stage for life-long host-microbial interaction and immune homeostasis. J. Immunol. 198, 557-563 (2017).

16. Liu, P. et al. The immunologic status of newborns born to SARS-CoV-2infected mothers in Wuhan, China. J. Allergy Clin. Immunol. 146, 101-109 (2020).

17. Garcia-Flores, V. et al. Maternal-fetal immune responses in pregnant women infected with SARS-CoV-2. Preprint at https://doi.org/10.21203/rs.3.rs362886/v1 (2021). 
18. Pickering, S. et al. Comparative assessment of multiple COVID-19 serological technologies supports continued evaluation of point-of-care lateral flow assays in hospital and community healthcare settings. PLoS Pathog. 16, e1008817 (2020).

19. Lu-Culligan, A. et al. Maternal respiratory SARS-CoV-2 infection in pregnancy is associated with a robust inflammatory response at the maternal-fetal interface. Med (NY) 2, 591-610 (2021).

20. Laing, A. G. et al. A dynamic COVID-19 immune signature includes associations with poor prognosis. Nat. Med. 26, 1623-1635 (2020).

21. Arunachalam, P. S. et al. Systems biological assessment of immunity to mild versus severe COVID-19 infection in humans. Science 369, 1210-1220 (2020).

22. Tornblom, S. A. et al. mRNA expression and localization of bNOS, eNOS and iNOS in human cervix at preterm and term labour. Reprod. Biol. Endocrinol. 3, 33 (2005).

23. Flannery, D. D. et al. Assessment of maternal and neonatal cord blood SARS-CoV-2 antibodies and placental transfer ratios. JAMA Pediatr. 175 594-600 (2021).

24. Martinez, D. R. et al. Fc characteristics mediate selective placental transfer of IgG in HIV-infected women. Cell 178, 190-201 (2019).

25. Goncalves, G. et al. Transplacental transfer of measles and total IgG. Epidemiol. Infect. 122, 273-279 (1999).

26. Edlow, A. G. et al. Assessment of maternal and neonatal SARS-CoV-2 viral load, transplacental antibody transfer, and placental pathology in pregnancies during the COVID-19 pandemic. JAMA Netw. Open 3, e2030455 (2020).

27. Atyeo, C. et al. Compromised SARS-CoV-2-specific placental antibody transfer. Cell 184, 628-642 (2021).

28. Bordt, E. A. et al. Sexually dimorphic placental responses to maternal SARS-CoV-2 infection. Preprint at https://doi.org/10.1101/2021.03.29.437516 (2021)

29. Beharier, O. et al. Efficient maternal to neonatal transfer of antibodies against SARS-CoV-2 and BNT162b2 mRNA COVID-19 vaccine. J. Clin. Invest. 131, e150319 (2021).

30. Gleditsch, D. D. et al. Maternal inflammation modulates infant immune response patterns to viral lung challenge in a murine model. Pediatr. Res. 76, 33-40 (2014).

31. Apostol, A. C., Jensen, K. D. C. \& Beaudin, A. E. Training the fetal immune system through maternal inflammation-a layered hygiene hypothesis. Front. Immunol. 11, 123 (2020).

32. Bilbo, S. D. \& Schwarz, J. M. Early-life programming of later-life brain and behavior: a critical role for the immune system. Front. Behav. Neurosci. 3, 14 (2009).

33. Acquah, J. K., Dahal, R. \& Sloan, F. A. 1918 influenza pandemic: in utero exposure in the United States and long-term impact on hospitalizations. Am. J. Public Health 107, 1477-1483 (2017)
34. Lu, W. et al. Early immune responses and prognostic factors in children with COVID-19: a single-center retrospective analysis. BMC Pediatr. 21, 181 (2021).

35. Sherer, M. L. et al. Pregnancy alters interleukin-1 beta expression and antiviral antibody responses during severe acute respiratory syndrome coronavirus 2 infection. Am. J. Obstet. Gynecol. 225, 301.e1-301.e14 (2021).

36. Lohman-Payne, B. et al. HIV-exposed uninfected infants: elevated cord blood interleukin 8 (IL-8) is significantly associated with maternal HIV infection and systemic IL-8 in a Kenyan cohort. Clin. Transl. Med 7, 26 (2018).

37. Reuschel, E. et al. Perinatal gram-positive bacteria exposure elicits distinct cytokine responses in vitro. Int. J. Mol. Sci. 22, 332 (2020).

38. Jouan, Y. et al. Phenotypical and functional alteration of unconventional T cells in severe COVID-19 patients. J. Exp. Med. 217, e20200872 (2020).

39. Kuri-Cervantes, L. et al. Comprehensive mapping of immune perturbations associated with severe COVID-19. Sci. Immunol. 5, eabd7114 (2020).

40. Gibbons, D. et al. Interleukin-8 (CXCL8) production is a signatory T cell effector function of human newborn infants. Nat. Med. 20, 1206-1210 (2014).

41. White, G. P., Watt, P. M., Holt, B. J. \& Holt, P. G. Differential patterns of methylation of the IFN- $\gamma$ promoter at $\mathrm{CpG}$ and non-CpG sites underlie differences in IFN- $\gamma$ gene expression between human neonatal and adult CD45RO $^{-}$T cells. J. Immunol. 168, 2820-2827 (2002).

42. Tang, W. Y. et al. Maternal exposure to polycyclic aromatic hydrocarbons and $5^{\prime}$-CpG methylation of interferon- $\gamma$ in cord white blood cells. Environ. Health Perspect. 120, 1195-1200 (2012).

43. van Esch, B. et al. The impact of milk and its components on epigenetic programming of immune function in early life and beyond: implications for allergy and asthma. Front Immunol. 11, 2141 (2020).

44. Hong, M. et al. Trained immunity in newborn infants of HBV-infected mothers. Nat. Commun. 6, 6588 (2015).

45. Garcia-Knight, M. A. et al. Altered memory T cell responses to Bacillus Calmette-Guerin and tetanus toxoid vaccination and altered cytokine responses to polyclonal stimulation in HIV-exposed uninfected Kenyan infants. PLoS ONE 10, e0143043 (2015).

46. Mishra, A. et al. Microbial exposure during early human development primes fetal immune cells. Cell 184, 3394-3409 (2021).

47. Fajnzylber, J. et al. SARS-CoV-2 viral load is associated with increased disease severity and mortality. Nat. Commun. 11, 5493 (2020).

48. Kappanayil, M. et al. Multisystem inflammatory syndrome in a neonate, temporally associated with prenatal exposure to SARS-CoV-2: a case report. Lancet Child Adolesc. Health 5, 304-308 (2021).

Publisher's note Springer Nature remains neutral with regard to jurisdictional claims in published maps and institutional affiliations.

(C) The Author(s), under exclusive licence to Springer Nature America, Inc. 2021 


\section{Methods}

Study design and human material. This study complies with all ethical regulation and was approved by the relevant research ethics committee (REC) as subsequently described. Umbilical cord blood (and paired maternal peripheral blood) was collected over the COVID-19 pandemic (28 May 2020-1 March 2021) at the time of birth from neonates born to mothers who were exposed to SARS-CoV-2 (SE) attending the maternity unit at Guy's and St Thomas' NHS Foundation Trust (GSTT), London, in heparinized blood tubes at the time of birth to investigate the immune status (REC approval no. 19/SC/0232, Infectious Diseases Bio-BankGuys \& St Thomas's Hospital, South Central, Hampshire B REC). Written informed consent was obtained from all participants before study entry. Mother-neonate dyads were categorized into recent or ongoing $(\mathrm{R} / \mathrm{O})$ and recovered $(\mathrm{R})$ groups, according to the number of days before birth that the mother received a positive status from a SARS-CoV-2 nasopharyngeal swab (R/O, $<14 \mathrm{~d} ; \mathrm{R},>14 \mathrm{~d})$. Maternal and neonatal SE plasma samples were used for antibody and cytokine testing. Neonatal cell samples were used in flow cytometry experiments to measure neonatal cell populations and some $(n=8)$ were used in SARS-CoV-2-reactive cell testing experiments along with their paired mother cell samples $(n=8)$. Umbilical cord blood was also collected at the time of birth from neonates born to healthy mothers at GSTT before the COVID-19 pandemic (until 1 Jan 2020), hence their mothers were not infected with SARS-CoV-2 at any time during their pregnancy, termed the non-SARS-CoV-2 exposed (NSE) group (REC approval no. 17/ LO/0641, London-Camberwell St. Giles REC). NSE samples were used as a control group for the flow cytometry. An additional group of NSE samples $(n=5)$ were used as a control group for the SARS-CoV-2-reactive cell testing experiments. The clinical details of the groups can be found in Table 1 .

Isolation of cord blood mononuclear cells and plasma. CBMCs were isolated from all neonate groups. Peripheral blood mononuclear cells (PBMCs) were also isolated from eight SE mothers for use in SARS-CoV-2-specific FluoroSpot assays. Neat plasma was also isolated from the SE neonates and their paired mothers. More specifically, for the paired maternal peripheral blood samples, whole blood was centrifuged at 2,000 g for $10 \mathrm{~min}$ at room temperature, and plasma was collected from the upper layer and stored at $-80^{\circ} \mathrm{C}$ in polypropylene tubes. For the neonatal samples, cord blood was layered onto Ficoll (GE Healthcare), within a 15-ml polypropylene conical tube, and centrifuged at $800 \mathrm{~g}$ for $15 \mathrm{~min}$ (break OFF) at room temperature. Plasma was then collected from the top fraction and stored at $-80^{\circ} \mathrm{C}$ in polypropylene tubes. The CBMC layer was isolated and subsequently washed twice with pre-warmed base medium (RPMI-1640+L-glutamine, Gibco), and then complete medium (CM; RPMI-1640 + 330 L-glutamine; $10 \%$ heat-inactivated FBS; $1 \%$ penicillin-streptomycin, Gibco), under centrifugation at $300 \mathrm{~g}$ for $5 \mathrm{~min}$ at room temperature. For maternal PBMC isolation, the blood was diluted with half volume in PBS and layered onto Leucosep (Greiner) tubes containing $15 \mathrm{ml} \mathrm{Ficoll.} \mathrm{The}$ PBMC layer was then isolated and washed twice with PBS under centrifugation at $300 \mathrm{~g}$ for $5 \mathrm{~min}$ at room temperature. The cell pellets were then frozen in Cryostor CS10 (Sigma) within polypropylene cryovials before proceeding with flow cytometry.

Polyclonal stimulation. Before staining the CBMCs for flow cytometry immunophenotyping with panel 4 (Supplementary Table 1), cells were thawed and plated in 96-well round-bottom plates (Corning) within $200 \mu \mathrm{l} \mathrm{CM}$ containing PMA (10 $\mathrm{ng} \mathrm{ml}^{-1}$; Sigma), ionomycin ( $1 \mathrm{~g} \mathrm{ml}^{-1}$; Sigma), brefeldin A (20 $\mathrm{ng} \mathrm{ml}^{-1}$; Sigma) and monensin solution (1X; BioLegend). A brefeldin A-only and monensin-only control was also plated for each neonate. Cells were incubated at $37^{\circ} \mathrm{C}$ for $4 \mathrm{~h}$ in the Biosafety level 3 (BSL-3) containment laboratory, in accordance with the King's College London safety rules before proceeding with flow cytometry staining. Cells were removed from BSL-3 once they were fixed with Cell Fix (1×; BD) for a minimum of $10 \mathrm{~min}$

Flow cytometry staining and acquisition. CBMCs were thawed and plated in 96-well round-bottom plates before staining in one of four panels (Supplementary Table 1) assessing the following immune cell populations (panel $1, \mathrm{~T}$ cell naive/ memory status; panel 2, myeloid and B cells; panel 3, T and NK cell activation status; panel $4, \mathrm{~T}$ and NK cytokine potential). All four panels contained surface-marker staining, and panel 1 and 4 also contained intracellular staining. All the following wash steps were performed under 2,000g, for $1 \mathrm{~min}$ at room temperature. For each panel, cells were washed with $100 \mu \mathrm{l}$ Dulbecco's PBS (1×; Gibco) and resuspended in $100 \mu \mathrm{l}$ PBS, containing Zombie NIR Fixable Viability dye (1:1,000 dilution; BioLegend), with the addition of TCR V81-FITC (TS8.2; Thermo Fisher) in panel 1 , for $15 \mathrm{~min}$ in the dark at $4^{\circ} \mathrm{C}$. Cells were then washed with $150 \mu \mathrm{l}$ eBioscience FOXP3 fixation/permeabilization buffer (Invitrogen), for panel 1, or FACS buffer (PBS, $0.5 \%$ heat-inactivated FBS, $2 \mathrm{mM}$ EDTA, Invitrogen), for panels $2-4$. The wash step was repeated with $200 \mu \mathrm{l}$ volume. Cells were then resuspended in $100 \mu \mathrm{l}$ eBioscience FOXP3 permeabilization buffer (FPB; Invitrogen), for panel 1, or $50 \mu \mathrm{l}$ surface antibody cocktail within FACS buffer, for panels 2-4, for 30 min (panels 2-3) or $20 \mathrm{~min}$ (panel 4 ), at $4^{\circ} \mathrm{C}$ in the dark. Cells were subsequently washed in $100 \mu \mathrm{l}$ FPB (panel 1) or $150 \mu$ l FACS buffer (panels 2-4), and again with $200 \mu$ l volume before resuspending in $50 \mu$ l antibody cocktail in FPB (panel 1), or $100 \mu$ l Cell Fix (1×; panels 2-4) for $30 \mathrm{~min}$ (panels 1-3) or until ICS (panel 4) at $4{ }^{\circ} \mathrm{C}$ in the dark. Panels 1-3 were then washed twice in $100 \mu \mathrm{l} \mathrm{FPB} \mathrm{(panel} \mathrm{1)} \mathrm{or} \mathrm{FACS} \mathrm{buffer} \mathrm{(panels}$
$2-3$ ) and resuspended in $200 \mu \mathrm{l}$ FACS buffer until acquisition. For the panel 4 ICS, cells were centrifuged at $2,000 \mathrm{~g}$, for $1 \mathrm{~min}$ at room temperature, and resuspended in $50 \mu 1$ ICS antibody cocktail within Permeabilization Wash Buffer (1×; PWB; BioLegend) for $30 \mathrm{~min}$ in the dark at room temperature. Cells were washed twice in $150 \mu \mathrm{l}$ PWB, followed by $200 \mu \mathrm{l}$ FACS buffer before resuspending the cells in FACS buffer for acquisition in a high-throughput sampler on a four-laser LSR Fortessa (BD) using FACSDiva (v8, BD), at a flow rate of $1 \mu \mathrm{s}^{-1}$.

Flow cytometry data analysis. Raw FCS files were analyzed using FlowJo (v10.6.2, BD), and the gating strategies are included in Extended Data Fig. 3. As an internal control, the same adult sample was run alongside each flow cytometry experiment for consistency and to aid setting gates. Data were cleaned up by gating on the Time parameter to ensure that only cells going through a constant flow stream were analyzed, and cell populations were excluded from downstream analysis if the event count in the parent population was $<30$.

SARS-CoV-2-specific antibody testing. ELISA assays were conducted as previously described $\mathrm{d}^{18,49}$. All plasma samples were heat inactivated at $56^{\circ} \mathrm{C}$ for $30 \mathrm{~min}$ before use. High-binding ELISA plates (Corning, 3690) were coated with antigen (nuclear protein $(\mathrm{N})$, spike glycoprotein (S) or the RBD at $3 \mu \mathrm{g} \mathrm{ml}^{-1}(25 \mu \mathrm{l}$ per well) in PBS, either overnight at $4^{\circ} \mathrm{C}$ or for $2 \mathrm{~h}$ at $37^{\circ} \mathrm{C}$. Wells were washed with PBS-T (PBS with $0.05 \%$ Tween-20) and then blocked with $100 \mu$ of $5 \%$ milk in PBS-T for $1 \mathrm{~h}$ at room temperature. Wells were emptied and serial dilutions of plasma (starting at 1:25, fivefold dilution) were added and incubated for $2 \mathrm{~h}$ at room temperature. Control reagents included CR3009 $\left(2 \mu \mathrm{g} \mathrm{ml}^{-1} ; \mathrm{N}\right.$-specific monoclonal antibody), CR3022 (0.2 $\mathrm{\mu g} \mathrm{ml}^{-1}$; S-specific monoclonal antibody), negative-control plasma (1:25 dilution), positive-control plasma (1:50 dilution) and blank wells. Wells were washed with PBS-T. Secondary antibody was added and incubated for $1 \mathrm{~h}$ at room temperature. IgG was detected using goat anti-human Fc-AP (1:1,000 dilution; Jackson, 109-055-098), and wells were washed with PBS-T. AP substrate (Sigma) was added and plates read at $405 \mathrm{~nm}$. IgM was detected using goat anti-human IgM-HRP (1:1,000 dilution; Sigma, A6907) and wells were washed with PBS-T. One-step 3,3',5,5'-tetramethylbenzidine substrate (Thermo Fisher Scientific) was added and quenched with $2 \mathrm{M} \mathrm{H}_{2} \mathrm{SO}_{4}$ before reading at $450 \mathrm{~nm}$ (HRP). Samples were run in duplicate and peak fold-change values above background were plotted for each patient. IgG transfer ratios were calculated to display the difference in paired neonate and maternal levels (neonatal IgG peak fold change over background/maternal IgG peak fold change over background).

Quantification of plasma cytokines. Paired maternal and neonatal plasma were thawed and tested in the 13-plex LegendPlex Human Anti-Virus Response Panel kit (BioLegend), to quantify levels of IL-1 $\beta$, IL-6, TNF, IP-10, CXCL8, IL-12p70, IFN- $\alpha 2$, IFN- $\lambda 1$, IFN- $\lambda 2 / 3$, GM-CSF, IFN- $\beta$, IL-10 and IFN- $\gamma$. The assay was performed according to the manufacturer's instructions and was modified by diluting the kit reagents $2 \times$ (beads, detection antibodies and streptavidin-PE). All plasma samples were diluted $2 \times$ with assay buffer, and resulting sample concentrations were calculated according to the dilution factor. In brief, $25 \mu$ l diluted plasma or standard, and mixed beads (1:1 ratio) were added to each well (in V-bottom 96-well plates) and incubated for $1.5 \mathrm{~h}$. The samples were washed twice with wash buffer, incubated with $25 \mu \mathrm{l}$ detection antibodies for $1 \mathrm{~h}$ and then $25 \mu \mathrm{l}$ streptavidin-PE was added for a further $30 \mathrm{~min}$. The samples were then washed once with wash buffer, resuspended in $200 \mu \mathrm{l}$ wash buffer and acquired on a high-throughput sampler with a three-laser FACSCanto (BD). All incubation steps were performed under 600 r.p.m. on an orbital shaker at room temperature and protected from light. Data were cleaned up by excluding cytokines if the bead event count was $<90$, to ensure accurate analyses, performed using the Windows LegendPlex (v8.0, BioLegend) software.

IFN- $\boldsymbol{\gamma}^{+}$SARS-CoV-2-positive cell detection by FluoroSpot assay. Cryopreserved CBMCs and PBMCs from SE neonates and their pared mothers, respectively, were thawed and stimulated with SARS-CoV-2 peptide pools to test for IFN- $\gamma^{+}$-positive cells in FluoroSpot assays (Mabtech), adapted from Monin et al. ${ }^{50}$. Specifically, cells were stimulated with peptide pools spanning the SARS-CoV-2 Matrix and Nucleocapsid (M\&N; Miltenyi), N-terminal domain of the SARS-CoV-2 spike protein (S1; JPT Peptide Technologies) or C-terminal domain of the SARS-CoV-2 spike protein (S2; JPT Peptide Technologies). CBMCs from NSE neonates were also tested. All FluoroSpot reagents were from Mabtech unless otherwise specified and used according to the manufacturer's instructions. In brief, 96-well PVDF membrane plates were coated with anti-IFN- $\gamma$ monoclonal capture antibody $\left(1-\mathrm{D} 1 \mathrm{~K}, \mathrm{FSX}-01 \mathrm{~A}-1 ; 15 \mu \mathrm{g} \mathrm{ml}^{-1}\right)$ at $4^{\circ} \mathrm{C}$. Plates were washed $4 \times$ with $200 \mu \mathrm{l}$ cold PBS and blocked with $200 \mu \mathrm{l}$ medium for $1 \mathrm{~h}$ (RPMI-GlutaMAX (Gibco), 2\% penicillin, streptomycin and amphotericin B (Gibco) and 10\% human AB serum (Sigma). After thawing, cells were rested at room temperature for $1 \mathrm{~h}$ and plated at $1 \times 10^{5}$ or $2 \times 10^{5}$ cells per well in the coated plate at $100 \mu l$ final volume of medium containing M\&N (1:200 dilution), S1 (1:2,000 dilution) or S2 (1:2,000 dilution) for $14 \mathrm{~h}$ at $37^{\circ} \mathrm{C}$ in duplicate conditions. Dimethylsulfoxide was used as a negative control, and each individual sample was plated at an equal cell number per condition. The incubation was performed in the BSL-3 containment laboratory, in accordance with the King's College London safety rules before fixing and reading the plates. After incubation, plates were washed $4 \times$ with $200 \mu \mathrm{l}$ PBS before adding 
$100 \mu \mathrm{l}$ anti-IFN- $\gamma$ BAM monoclonal detection antibody (7-B6-1-BAM, FSX-01A-1; $1: 200$ dilution) in PBS- $0.1 \%$ BSA for $2 \mathrm{~h}$ at room temperature in the dark. Plates were washed as above, and $100 \mu \mathrm{l}$ anti-BAM-490 (FSX-01A-1; 1:200 dilution) in PBS- $0.1 \%$ BSA was added for $1 \mathrm{~h}$ at room temperature in the dark. The wash step was repeated and $50 \mu \mathrm{l}$ fluorescence enhancer was added for $10 \mathrm{~min}$ in the dark before removing the liquid and the plastic tray and drying the plates overnight. A total of $100 \mu \mathrm{l} 2 \%$ paraformaldehyde (Alfa Aesar) in PBS was then added for $30 \mathrm{~min}$. After fixing, the plates were dried and the number of IFN- $\gamma^{+}$spots per $10^{6}$ cells was detected and analyzed using AID iSpot Spectrum reader and EliSpot software (v8.0 Autoimmun Diagnostika). A threshold level of positivity was set at $>1 \mathrm{IFN}-\gamma^{+}$cells per well (equivalent to 2.5 cytokine-secreting cells per $10^{6}$ cells).

Statistical analyses. Analyzed flow cytometry populations, plasma cytokines and antibodies were imported into an Excel spreadsheet and analyzed in R (v4.0.3) to generate box plots, dimensionality reduction plots, Spearman correlation plots and heat maps. Clustered heat maps were performed on scaled and centered data using the heatmap. 2 function (gplots package), and clustered according to the Euclidean method. The corrplot package was used to generate Spearman correlation matrices, and only significant values $(P<0.05)$ are displayed. GraphPad Prism (v9.0) was also utilized to generate scatterplots for cytokines and the antibody heat map. All statistical tests were used to measure the differences between biologically distinct samples. Unadjusted $P$ values $\left({ }^{\star} P<0.05 ;{ }^{* *} P<0.01 ;{ }^{* *} P<0.001\right.$; ${ }^{* * * *} P<0.0001$ ) were assessed by the Kolmogorov-Smirnov test (to compare cytokine concentrations between the groups), two-sided Wilcoxon rank-sum tests (for immune cell populations between the groups) and two-sided paired Wilcoxon tests (between paired maternal and neonatal antibody/cytokine levels).

Reporting Summary. Further information on research design is available in the Nature Research Reporting Summary linked to this article.

\section{Data availability}

The raw FCS files generated during this study have been deposited in FlowRepository under accession code FR-FCM-Z46A. Source data are provided with this paper.

\section{Code availability}

Code was generated using the publicly available R (v4.0.3) packages: ggplot2, gplots, dplyr, dynutils, tidyr, viridis, ggrepel, ggfortify, Hmisc, Rtsne and corrplot.

\section{References}

49. Seow, J. et al. Longitudinal observation and decline of neutralizing antibody responses in the three months following SARS-CoV-2 infection in humans. Nat. Microbiol. 5, 1598-1607 (2020).

50. Monin, L. et al. Safety and immunogenicity of one versus two doses of the COVID-19 vaccine BNT162b2 for patients with cancer: interim analysis of a prospective observational study. Lancet Oncol. 22, 765-778 (2021).

\section{Acknowledgements}

We thank the mothers and their neonates for blood collection and all the midwives (GSTT) for sample collection. We also thank S. Kamdar and other staff involved with COVID-IP (King's College London) ${ }^{20}$ for some sample processing and T. Lechmere (King's College London) for assistance with ELISA assays. We thank I. Zlatareva (King's College London) for assistance with the cytokine multiplex analysis. We also acknowledge Evolve Biosystems for funding (to R.T. and D.G.) toward the NSE neonatal cord blood sample collection and the staff involved: N. Kelly, L. McMillan, S. Kheirallah and J. Mi; GSTT and King's College London). We thank Y. Wu (King's College London) for critical reading of the manuscript. S.G. is supported by an MRC-KCL Doctoral Training Partnership in Biomedical Sciences (MR/N013700/1); D.L.G. is supported by Action Medical Research (GN2790); M.C. is supported by the National Institute for Health Research (NIHR) BRC COVID-19 call; R.M.T. is supported by Tommy's (charity no. 1060508) and Borne (1167073); J.S. and K.J.D. are supported by a Kings Together Rapid COVID-19 call award and a Huo Family Foundation award; and E.P. and T.T. are supported by the T1DUK Immunotherapy Consortium funded by Diabetes UK (15/0005232). The research was also supported by the NIHR Biomedical Research Centre based at GSTT and King's College London (part of the King's Health Partners Academic Sciences Centre) and/or the NIHR Clinical Research Facility. The views expressed are those of the authors and not necessarily those of the NHS, the NIHR or the Department of Health and Social Care.

\section{Author contributions}

M.C. and C.M.: patient consent, study design and sample collection. S.G.: sample processing, flow cytometry and data analysis. S.G. and A.D.: panel design, multiplex cytokine testing and analysis. S.G., E.P. and T.T.: SARS-CoV-2-specific peptide FluoroSpot assays. J.S. and K.J.D.: antibody ELISA testing and analysis. D.G. and R.T.: manuscript writing and experimental design. All authors reviewed drafts of the manuscript before submission.

\section{Competing interests}

The authors declare no competing interests.

\section{Additional information}

Extended data is available for this paper at https://doi.org/10.1038/s41590-021-01049-2. Supplementary information The online version contains supplementary material available at https://doi.org/10.1038/s41590-021-01049-2.

Correspondence and requests for materials should be addressed to Deena L. Gibbons. Peer review Information: Nature Immunology thanks Daniele De Luca and the other, anonymous, reviewer(s) for their contribution to the peer review of this work. Editor recognition statement $\mathrm{N}$. Bernard was the primary editor on this article and managed its editorial process and peer review in collaboration with the rest of the editorial team.

Reprints and permissions information is available at www.nature.com/reprints. 

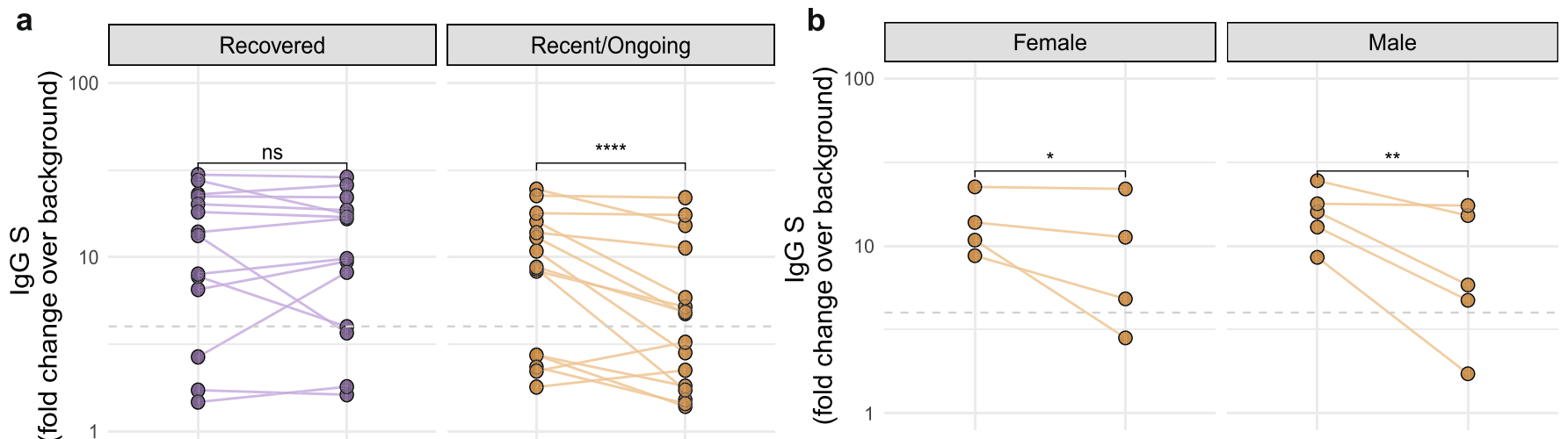

\section{Mother Neonate Mother Neonate}
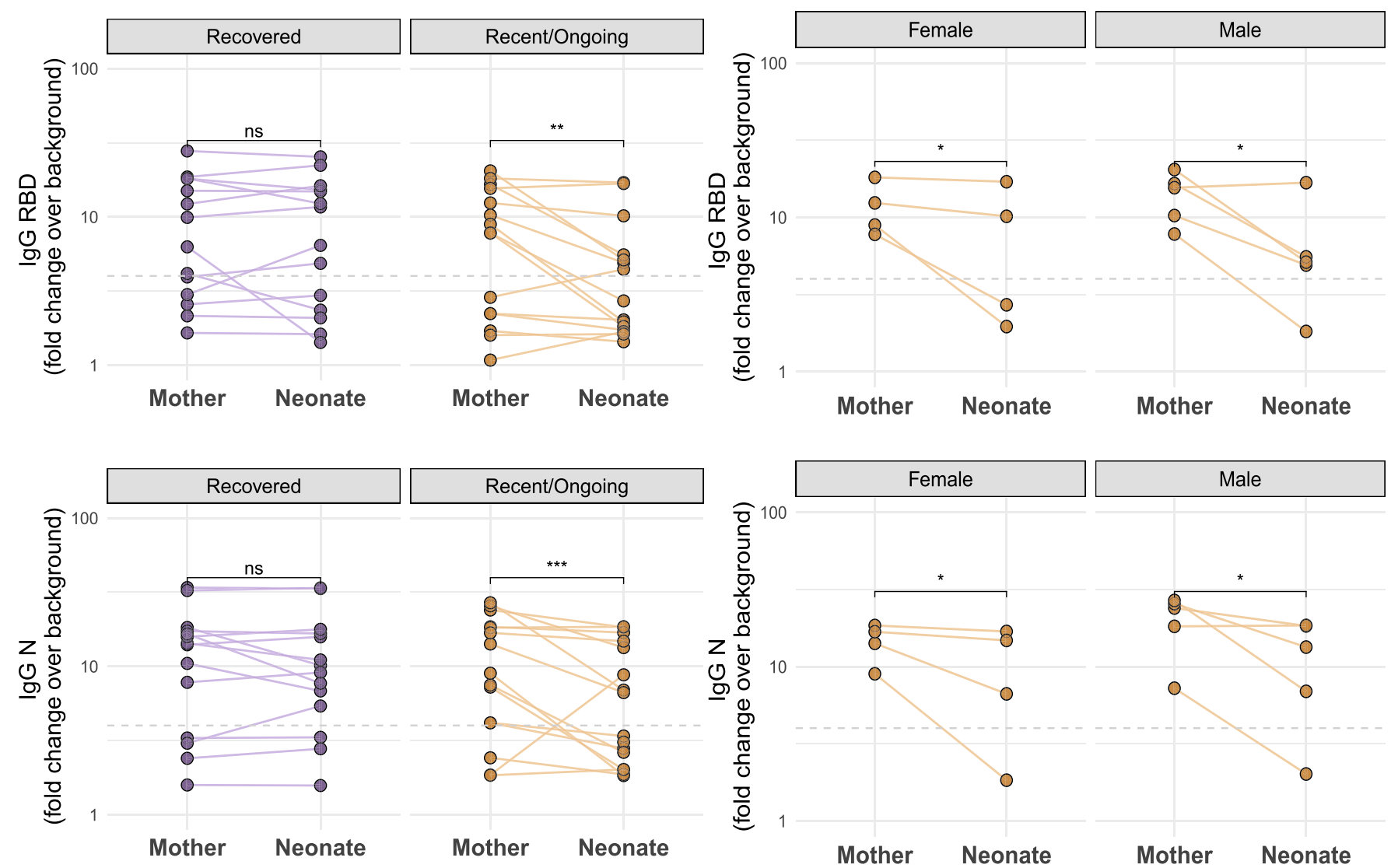

Extended Data Fig. 1 | Neonatal plasma IgG levels to SARS-CoV-2 antigens were lower than their mothers in the R/O group and no sex bias was observed. a, IgG levels measured from neonatal cord blood and paired maternal peripheral blood (taken at the time of delivery) against the S, RBD and N epitopes across the total R $(n=14)$ and R/O $(n=15)$ groups $(\mathbf{a})$, and in female $(n=13)$ and male $(n=16)$ neonates born to R/O seropositive mothers (b). Peak lgG/lgM levels (fold change over background) are plotted within the neonate-mother dyads. Seropositive lgG threshold (dashed grey line) $=$ $4 x$ fold change over background. Each line joins a neonate and their paired mother. P values were assessed by paired Wilcoxon tests (a,b). All tests were two-sided and all $p$ values $\left({ }^{\star} p<0.05 ;^{* \star} p<0.01 ;{ }^{* \star \star}<p<0.001 ;{ }^{* \star \star *} p<0.0001\right.$ ) were unadjusted. 


\section{Recovered}

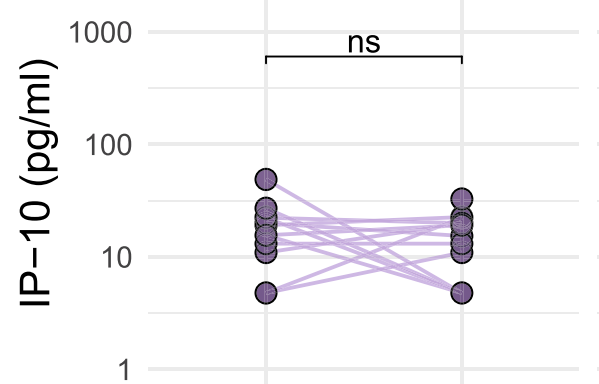

Mother Neonate
Recent/Ongoing

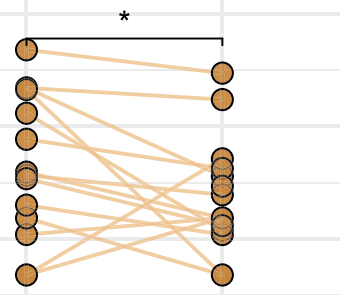

\section{Mother Neonate}

b
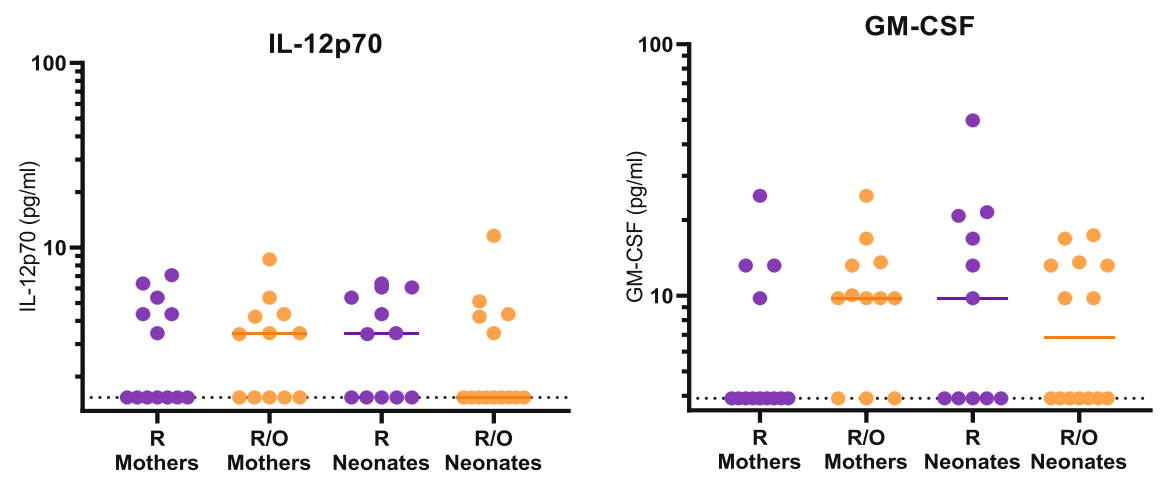

IFN- $\lambda 2 / 3$

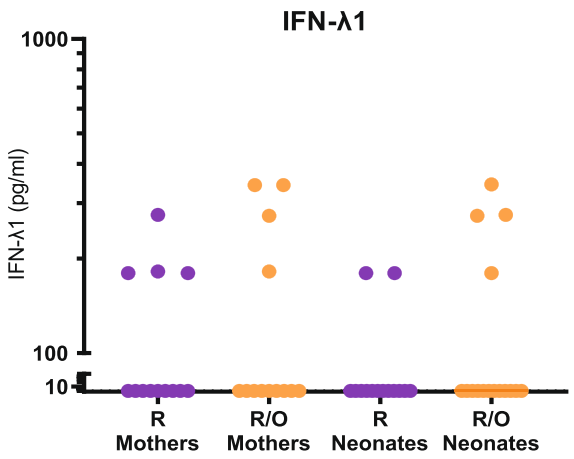

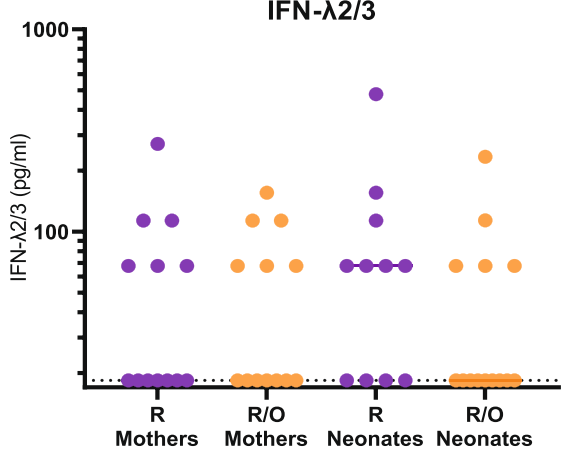

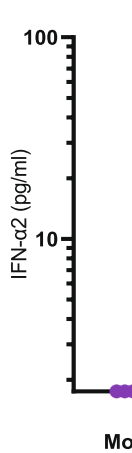

IFN- $\alpha 2$

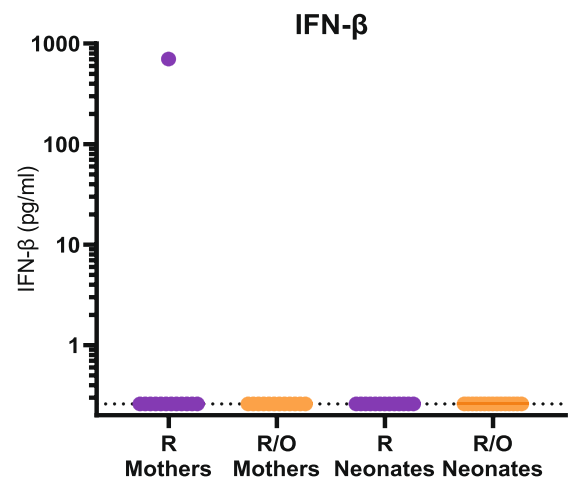

Extended Data Fig. 2 | Neonatal and paired maternal plasma cytokine concentrations within the R/O and R groups. a, Paired maternal and neonatal plasma IP-10 levels within the R $(n=12)$ and the $\mathrm{R} / \mathrm{O}(n=14)$ groups. Plasma was isolated from neonatal cord blood and maternal peripheral blood at the time of delivery. Each line joins a neonate and their paired mother. b, Plasma IL-12p70, GM-CSF, IFN- $\alpha 2$, IFN- $\lambda 1$, IFN- $\lambda 2 / 3$ and IFN- $\beta$ in the mothers $(\mathrm{R}-n=13, \mathrm{R} / \mathrm{O}-\mathrm{IFN}-\lambda 1$ IFN- $\lambda 2 / 3: n=13, \mathrm{GM}-\mathrm{CSF}, \mathrm{IL}-12 \mathrm{p} 70$, IFN- $\beta: n=12)$ and their neonates (R - IFN- $\lambda 1: n=14$, IFN- $\beta: n=13$, IL-12p70, IFN- $\alpha 2: n=12$, GM-CSF: $n=11$, R/O - IFN- $\lambda 1: n=16$, IFN- $\beta: n=15$, IFN- $\lambda 2 / 3$, GM-CSF, IL-12p70: $n=14$ ). TNF and IFN- $\gamma$ cytokines were not detected in any samples and are not presented. Horizontal dotted lines represent the minimum detectable concentrations. P values were assessed by paired Wilcoxon tests (a). All tests were two-sided and all $p$ values $\left({ }^{\star} p<0.05\right)$ were unadjusted. 
a Live, singlet lymphocytes gating (panel 1 and 4)

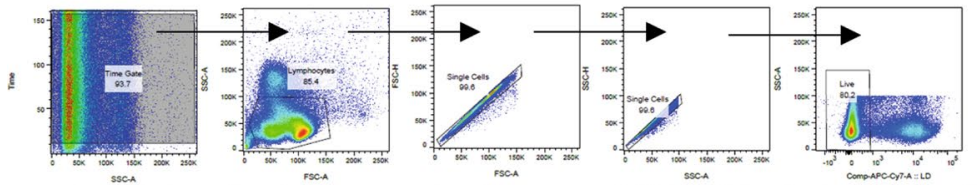

b Singlet cells gating (panel 2 and 3 )

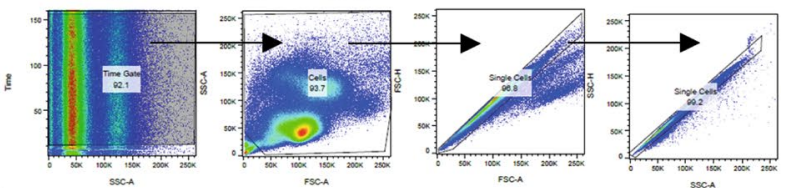

c Panel 1: naïve/memory T cell status, $V \delta 1 / V \delta 2 ~ Y \delta T$ cells and $T_{R E G S}$

Live singlet lymphocytes

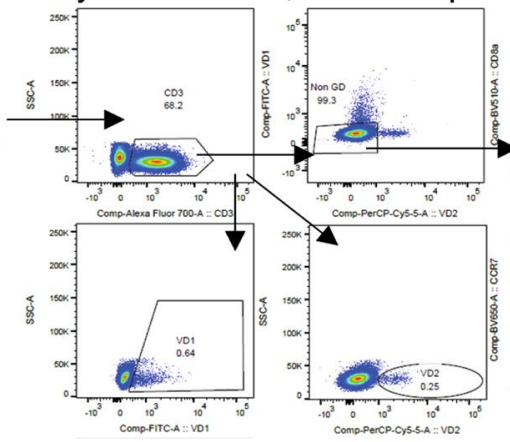

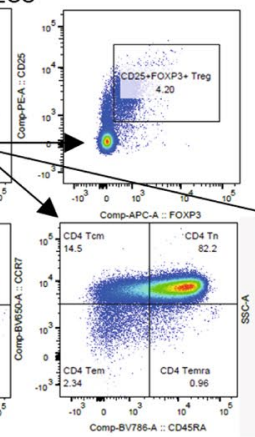

Ki67 expression

measured in all

$T$ cell populations

d Panel 2: Myeloid and B cell populations

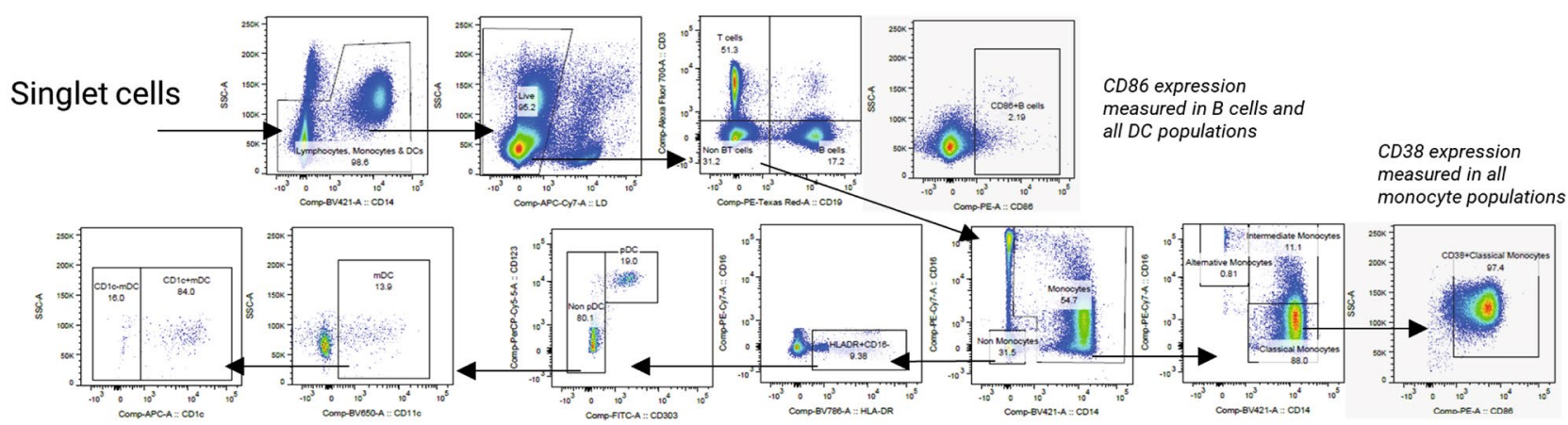

e Panel 3: T and NK/NKT cell activation status

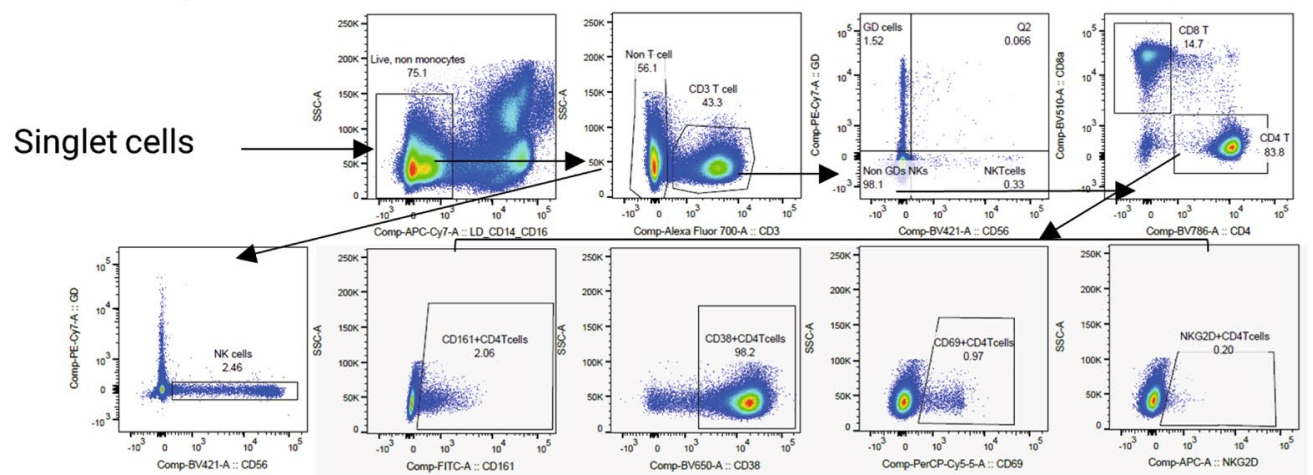

f Panel 4: T and NK/NKT cytokine functionality

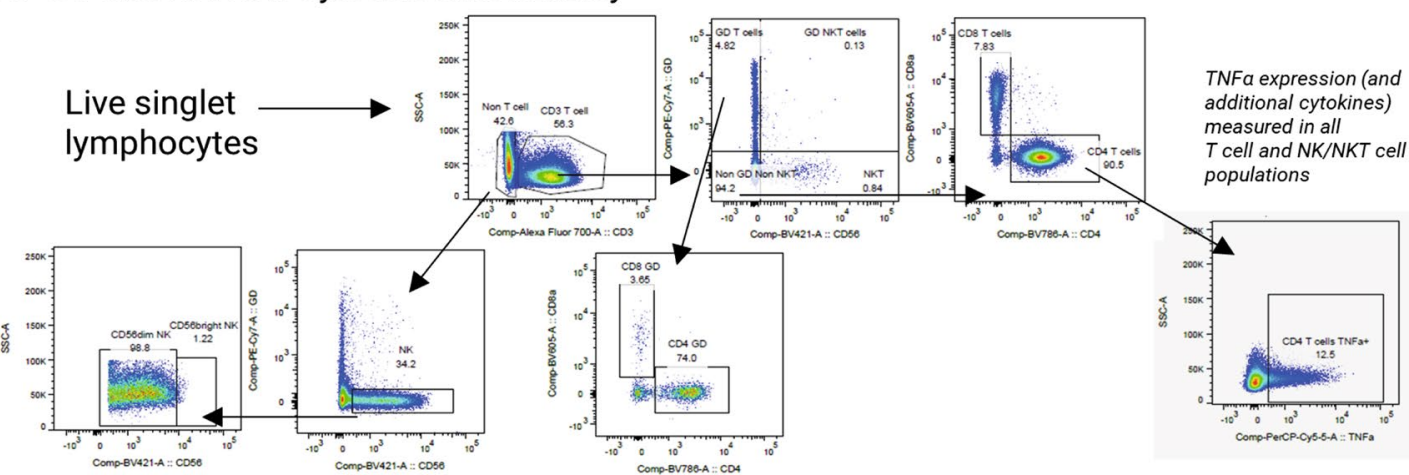

Extended Data Fig. 3 | See next page for caption. 
Extended Data Fig. 3 | Flow cytometry gating strategies for all four panels. a,b, Representative initial gating strategies for all 4 panels. Live singlet lymphocytes (a) or cells (b) were gated on Time vs SSC-A to ensure that only cells going through a constant flow stream were analysed. c-f, Representative gating strategies for panels 1-4 after initial gating. T cell naive/memory status, V $81 / \mathrm{V} \delta 2 \gamma \delta \mathrm{T}$ cell subtypes and $\mathrm{T}_{\mathrm{REGS}}$ (and their Ki67 expression) (c), B cell, dendritic cell (DC) an monocyte populations (and their CD86 or CD38 expression) (d), T cell and NK/NKT cell activation status (e) and T cell/NK/NKT cytokine functionality after polyclonal stimulation (f) were measured. Plots in grey boxes indicate populations that were measured in multiple cell types as described. 


\section{NATURE IMMUNOLOGY}

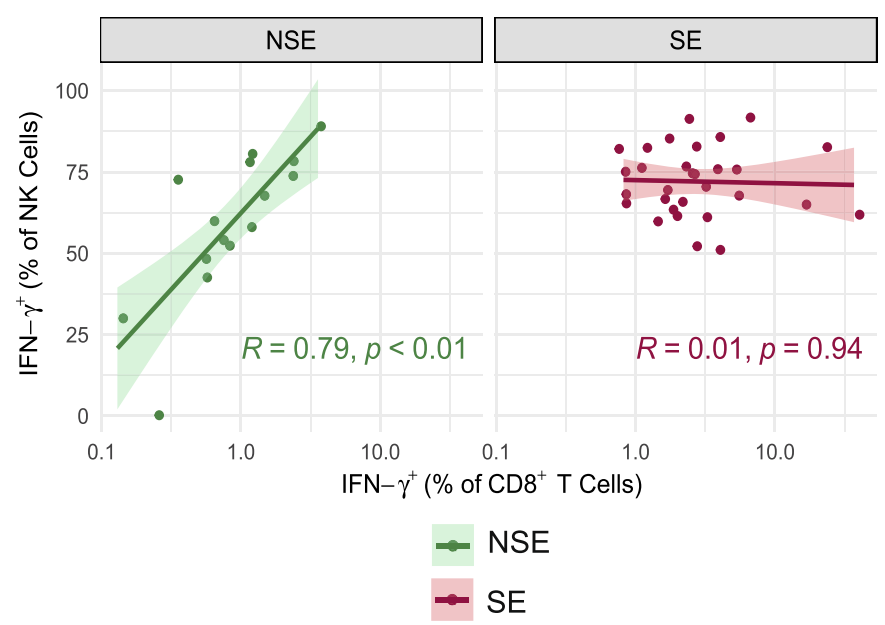

b

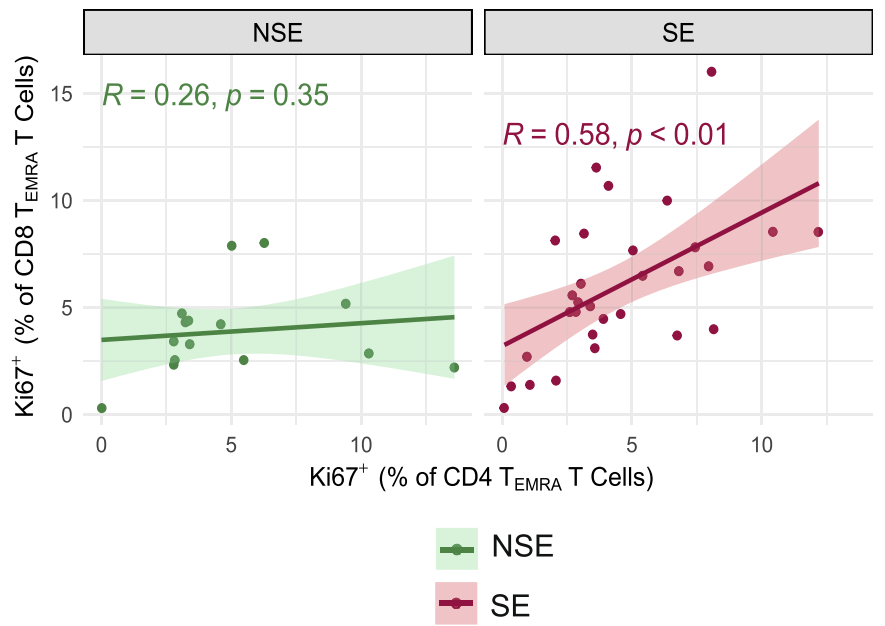

Extended Data Fig. 4 | Disrupted immune cell correlations in neonates within the SE group. a,b, Spearman correlation plots between IFN $\gamma+C D 8^{+} T$ cells and IFN $\gamma^{+} \mathrm{NK}$ cells $(\mathbf{a})$ and $\mathrm{Ki} 67{ }^{+} \mathrm{CD} 4{ }^{+} \mathrm{T}_{\mathrm{EMRA}}$ and $\mathrm{Ki} 67{ }^{+} \mathrm{CD} 8{ }^{+} \mathrm{T}_{\text {EMRA }}$ populations $(\mathbf{b})$ in the NSE $(n=15)$ and SE $(n=30)$ neonatal groups. Data indicates a generalised linear regression line with shaded $95 \%$ confidence intervals. Associated correlation coefficients and $p$ values are in green (NSE) or dark $\operatorname{red}(\mathrm{SE})$. 
GA

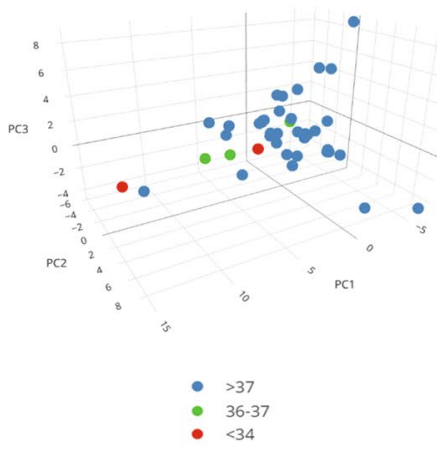

Maternal co-infection

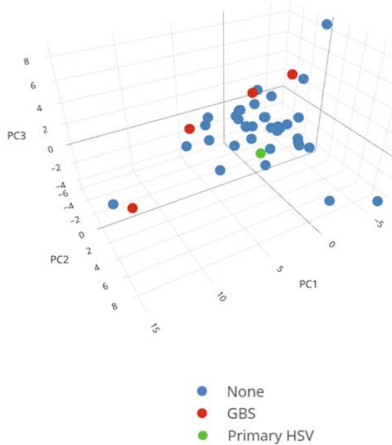

Sex of the neonate

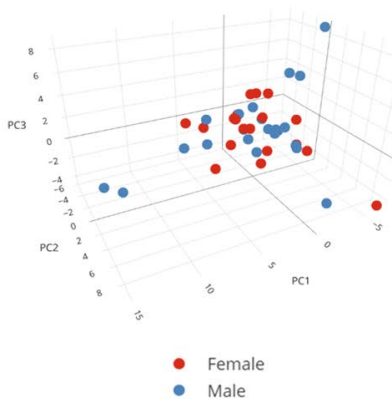

MOD

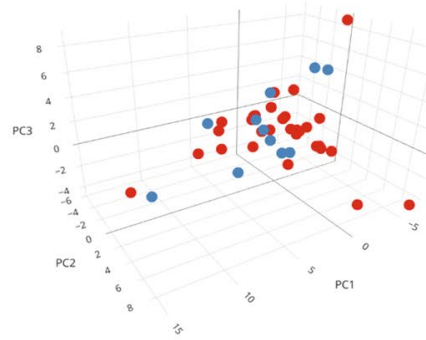

- Caesarean section (CS)

- Vaginal Delivery (VD)
Maternal ethnicity

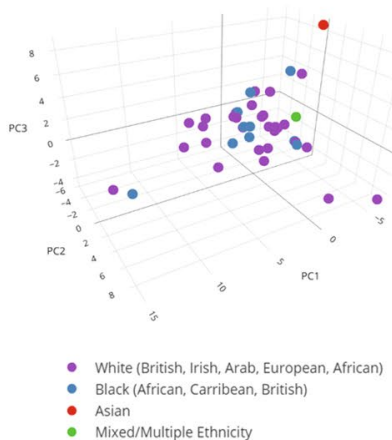

Maternal GDM/T2DM

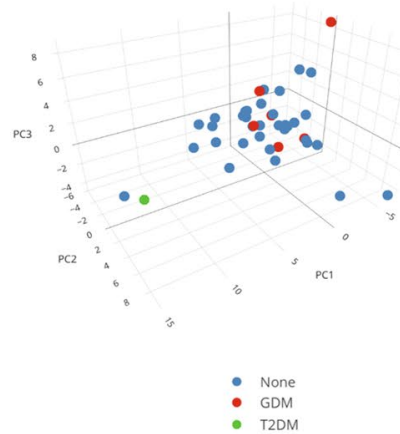

Chorioamnionitis

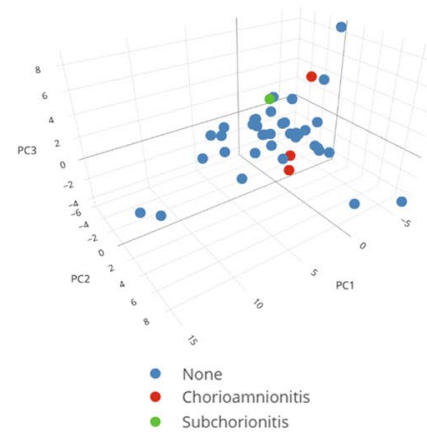

Extended Data Fig. 5 | PCA of the flow cytometry analysis coloured by alternative factors. 3-dimensional PCA dimensionality reduction of all flow cytometry data measured from CBMCs in the NSE $(n=13)$ and SE $(n=28)$ neonatal groups, coloured by alternative factors $(G A$, sex of the neonate, MOD, maternal ethnicity, maternal co-infection, maternal GDM/T2DM and chorioamnionitis). Chorioamnionitis was defined by placental histology testing: Individuals in the 'none' group were either not tested (as no placental pathology suspected) or normal placental histology upon testing. PC1, PC2, and PC3 explain $20.6 \%, 11.8 \%$ and $8.4 \%$ of the variance, respectively. 
a
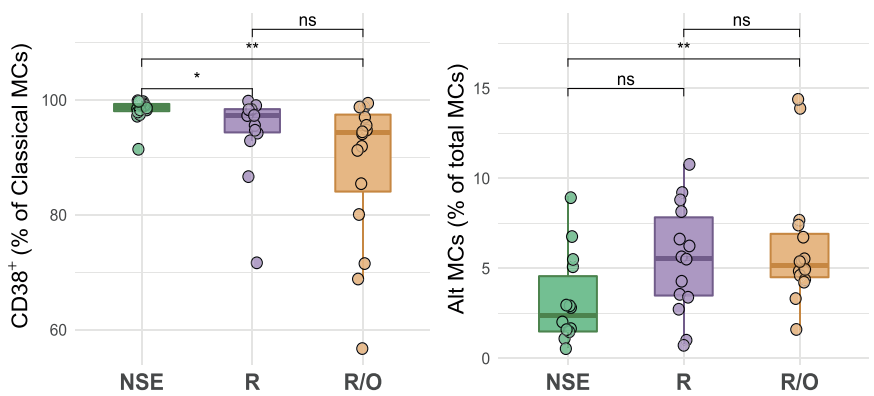

Non SARS-CoV-2 Exposed

Recovered

Recent/Ongoing

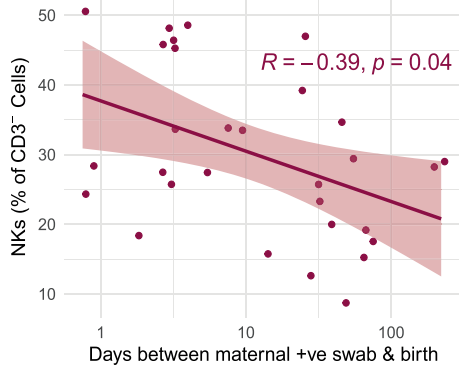

C

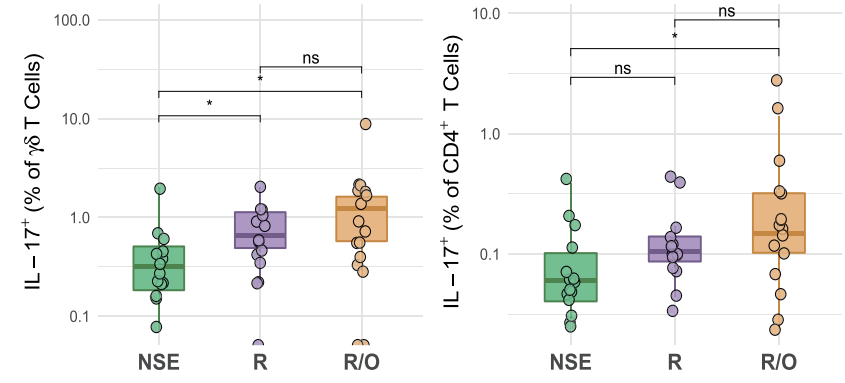

d

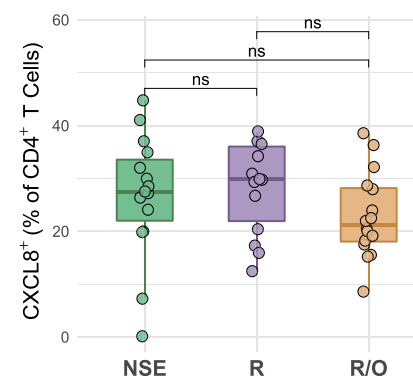

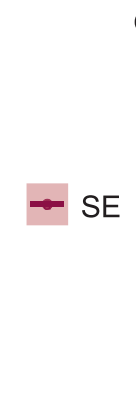
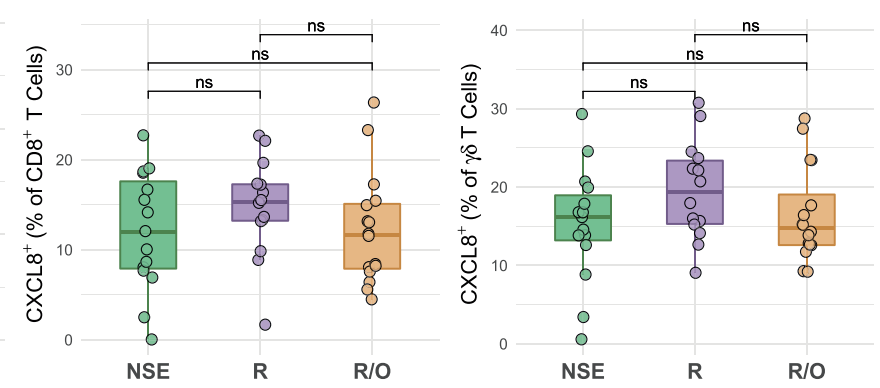

e

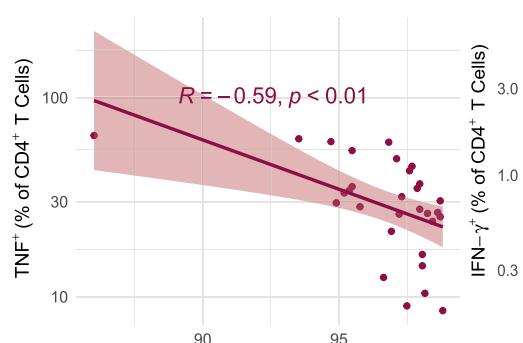

$\mathrm{CD}^{90} 8^{+}$(\% of $\mathrm{CD}^{+}{ }^{+} \mathrm{T}$ Cells)

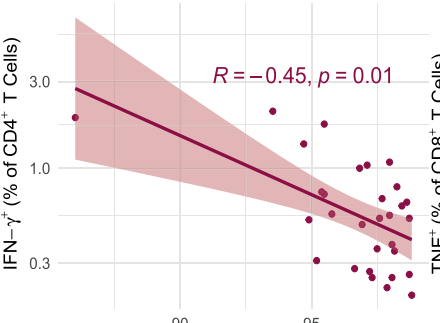

$\stackrel{90}{\mathrm{CD}^{+} 8^{+}}$(\% of CD4 $4^{+}$T Cells)

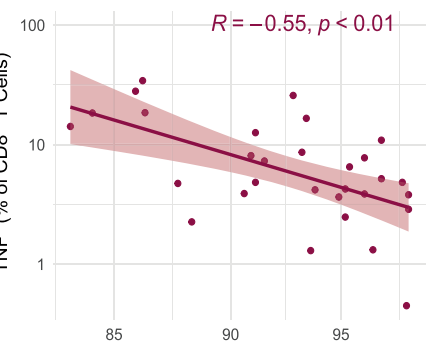

${ }^{85} 38^{+}$(\% of CD8 $8^{+}$T Cells)

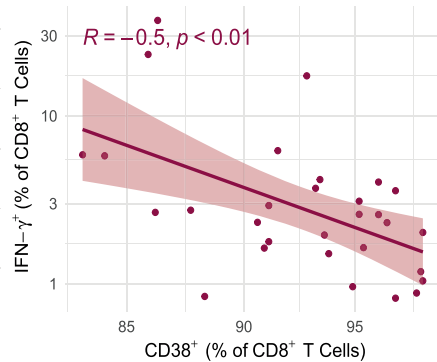

$\mathrm{CD}^{+} 8^{+}\left(\%\right.$ of $\mathrm{CD}^{+} \mathrm{T}$ Cells)

Extended Data Fig. 6 | Altered and unchanged neonatal cell populations within the SE group. a, Boxplots displaying the proportions of CD38 ${ }^{+}$classical monocytes and alternative monocytes in neonates within NSE $(n=14), \mathrm{R}(n=14)$ and R/O $(n=16)$ groups. $\mathbf{b}$, Spearman correlation plots in the SE group $(n=30)$ of neonatal proportions of NK cells and days between maternal positive nasopharyngeal swab and birth. $\mathbf{c}$, Boxplots displaying the proportions of $\mathrm{IL}-17^{+} \gamma \delta \mathrm{T}$ cells and CD4+ $\alpha \beta$ T cells in neonates within NSE $(n=15), \mathrm{R}(n=14)$ and R/O $(n=16)$ groups. $\mathbf{d}$, Boxplots displaying the proportions of CXCL8 ${ }^{+}$ $\mathrm{CD}^{+} \alpha \beta$ T cells, CD8 ${ }^{+} \alpha \beta$ T cells and $\gamma \delta$ T cells in neonates within NSE $(n=15), \mathrm{R}(n=14)$ and $\mathrm{R} / \mathrm{O}(n=16)$ groups. e, Spearman correlation plots in the SE group $(n=30)$ of neonatal proportions of $\mathrm{TNF}^{+}$or IFN $-\gamma^{+} \mathrm{CD} 4^{+} \mathrm{T}$ cells with CD38+CD4 ${ }^{+} \mathrm{T}$ cells $(\mathrm{LHS})$, and proportions of TNF+ or IFN- $\gamma^{+} \mathrm{CD} 8^{+} \mathrm{T}$ cells with $\mathrm{CD} 38^{+} \mathrm{CD} 8+\mathrm{T}$ cells (RHS). Spearman correlation data indicates generalised linear regression lines with shaded $95 \%$ confidence intervals. Associated Spearman correlation coefficients and $p$ values are in dark red. All boxplots follow standard Tukey representations; central line $=$ median, upper line $=75^{\text {th }}$ percentile; lower line $=25^{\text {th }}$ line; whiskers $=1.5^{\star} 75^{\text {th }} / 25^{\text {th }}$ percentile. $P$ values were assessed by unpaired Wilcoxon rank-sum tests $(a, c, d)$. All tests were two-sided and all $p$ values ( ${ }^{\star} p<0.05$ and $\left.{ }^{* \star} p<0.01\right)$ were unadjusted. 


\section{Reporting Summary}

Nature Research wishes to improve the reproducibility of the work that we publish. This form provides structure for consistency and transparency in reporting. For further information on Nature Research policies, see our Editorial Policies and the Editorial Policy Checklist.

\section{Statistics}

For all statistical analyses, confirm that the following items are present in the figure legend, table legend, main text, or Methods section.

$\mathrm{n} / \mathrm{a}$ Confirmed

$\bigotimes$ The exact sample size $(n)$ for each experimental group/condition, given as a discrete number and unit of measurement

\ A statement on whether measurements were taken from distinct samples or whether the same sample was measured repeatedly

The statistical test(s) used AND whether they are one- or two-sided

Only common tests should be described solely by name; describe more complex techniques in the Methods section.

\A description of all covariates tested

$\square$ A description of any assumptions or corrections, such as tests of normality and adjustment for multiple comparisons

$\checkmark$ A full description of the statistical parameters including central tendency (e.g. means) or other basic estimates (e.g. regression coefficient) AND variation (e.g. standard deviation) or associated estimates of uncertainty (e.g. confidence intervals)

For null hypothesis testing, the test statistic (e.g. $F, t, r$ ) with confidence intervals, effect sizes, degrees of freedom and $P$ value noted Give $P$ values as exact values whenever suitable.

Х $\square$ For Bayesian analysis, information on the choice of priors and Markov chain Monte Carlo settings

$\bigotimes \square$ For hierarchical and complex designs, identification of the appropriate level for tests and full reporting of outcomes

$\bigotimes \square$ Estimates of effect sizes (e.g. Cohen's $d$, Pearson's $r$ ), indicating how they were calculated

\section{Our web collection on statistics for biologists contains articles on many of the points above.}

\section{Software and code}

Policy information about availability of computer code

Data collection Clinical data were collected both manually and from the Badgernet database and exported into Microsoft Excel (v2108) after anonymisation. Flow cytometry cell immunophenotyping and multiplex cytokine data were collected using FACSDiva Software (v8, BD). Fluorospot data were collected on AID iSpot Spectrum reader (Autoimmun Diagnostika). Antibody data and analysed forms of all laboratory data were compiled into Microsoft Excel (v2108).

Data analysis FlowJo (v10.6.2, Treestar), GraphPad Prism (v9), LEGENDplex ${ }^{\text {TM }}$ Data Analysis (v8, BioLegend), EliSpot software (v8.0, Autoimmun Diagnostika). and R (v4.0.3) using the packages: ggplot2 (3.3.3), gplots (3.1.1), dplyr (1.0.2), dynutils (1.0.5), tidyr (1.1.2), viridis (0.5.1), ggrepel (0.9.0), ggfortify (0.4.11), Hmisc (4.4-2), Rtsne (0.15) and corrplot (0.84).

For manuscripts utilizing custom algorithms or software that are central to the research but not yet described in published literature, software must be made available to editors and

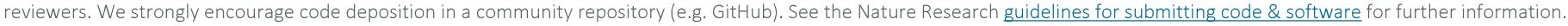

\section{Data}

Policy information about availability of data

All manuscripts must include a data availability statement. This statement should provide the following information, where applicable:

- Accession codes, unique identifiers, or web links for publicly available datasets

- A list of figures that have associated raw data

- A description of any restrictions on data availability

All figures have associated raw data. The raw FCS files generated during this study will be publicly available on flowrepository.org (Accession ID: FR-FCM-Z46A) before publication. 
Please select the one below that is the best fit for your research. If you are not sure, read the appropriate sections before making your selection.

\section{Life sciences study design}

All studies must disclose on these points even when the disclosure is negative.

Sample size Sample size calculations were not performed, due to the unprecedented nature of the COVID-19 pandemic and due to a lack of preexisting data. The sample size was determined on the basis of the number of infants/mothers presenting at the maternity clinic in GSTT (London) and consenting under informed consent to the study, during the COVID-19 waves.

Data exclusions For flow cytometry work, a parental gate threshold event number was set at 30, in that all daughter gate populations were excluded if the parent gate had <30 events, to ensure accurate analyses. For legendplex multiplex work, some of the 13 cytokines for some samples were excluded as the bead population event count was $<90$, and it is recommended by the manufacturer (Biolegend) to exclude low bead population samples, to ensure accurate analyses. The $n$ numbers for each plot are clearly stated in the figure legends.

\begin{tabular}{l|l} 
Replication & Replication was not performed as this was not possible from small volumes of unique human sample material.
\end{tabular}

Randomization Mothers with a positive nasopharengeal swab for SARS-CoV-2 (at any time in their pregnancy) were approached for the study and therefore allocated into the SE group. There was no other pre-selection and all demographics were eligible. Only after analysis were the groups then subdivided into those with recent infection ( $<14$ days before birth) and those with recovered infection ( $>14$ days before birth). This was the only basis for grouping and it was otherwise random. The NSE cohort were collected prior to the pandemic and were therefore guaranteed as non SARS-CoV-2 exposed (NSE) and again all mothers were approached to participate and all demographics were eligible. We did examine potential covariates between the groups which is shown in Extended Data Fig. 5.

Blinding Blinding of patient recruitment was not possible with the clinical team as participants were specifically recruited based on their COVID-19 status. Allocation of the SARS-CoV-2 exposed cohort into the recent/ongoing and recovered groups was done post-analysis (as explained above), thus the experimental researchers were blinded, although due to health and safety restrictions samples had to be identified as SE to the researchers for processing purposes. Clinical details were integrated into the study only after analyses were performed.

\section{Reporting for specific materials, systems and methods}

We require information from authors about some types of materials, experimental systems and methods used in many studies. Here, indicate whether each material, system or method listed is relevant to your study. If you are not sure if a list item applies to your research, read the appropriate section before selecting a response.

\begin{tabular}{l|ll} 
Materials \& experimental systems & Methods \\
\hline n/a & Involved in the study & \\
\hline & $\bigotimes$ Antibodies & $\square$ Eukaryotic cell lines \\
$\square$ & $\square$ Palaeontology and archaeology \\
$\square$ & $\square$ Animals and other organisms \\
$\searrow$ & $\square$ Clinical data \\
$\searrow$ & $\square$ Dual use research of concern
\end{tabular}



used in flow cytometry experiments in the format of antibody (clone, cat number, supplier, dilution). Panel 1: anti-CD45RA BV785 (HI100, 304140, BioLegend, 1:100), anti-CCR7 BV650 (GO43H7, 353234, BioLegend, 1:50), anti-CD8a BV510 (RPA-T8, 301048, BioLegend, 1:400), anti-Ki-67 BV421 (Ki-67, 350506, BioLegend, 1:50), anti-CD4 Pe-Cy7 (OKT4, 317414, BioLegend, 1:50), anti-CD25 PE (M-A251, 356104, BioLegend, 1:50), anti-TCR Vd2 PerCP (B6, 331410, BioLegend, 1:50), anti-TCR Vd1 FITC (TS8.2, TCR2730, Thermo Scientific, 1:100), anti-FOXP3 AF647 (259D, 320214, BioLegend, 1:50), anti-CD3 AF700 (OKT3, 317340, BioLegend,1:200). Panel 2: anti-HLA-DR BV785 (L234, 307642, BioLegend, 1:100), anti-CD11C BV650 (3.9, 301638. BioLegend, 1:50), anti-CD40 BV510 (5C3, 334330, BioLegend, 1:100), anti-CD14 BV421 (M5E2, 301830, BioLegend, 1:100), anti-CD16 Pe-Cy7 (3G8, 302016, BioLegend, 1:200), anti-CD19 PE-Dazzle 594 (HIB19, 302252, BioLegend, 1:100), anti-CD86 PE (IT2.2, 305406, BioLegend, 1:100), anti-CD123 PerCP-Cy5.5 (6H6, 306016, BioLegend, 1:50), anti-CD303 FITC (201A, 348208, BioLegend, 1:100), anti-CD1C (BCDA-1) APC (L161, 331524, BioLegend, 1:100), anti-CD3 AF700 (OKT3, 317340, BioLegend, 1:200). Panel 3: anti-CD4 BV785 (OKT4, 317442, BioLegend, 1:50), anti-CD38 BV650 (HB-7, 356620, BioLegend, 1:100), anti-CD8a BV510 (RPA-T8, 301048, BioLegend, 1:400), anti-CD56 BV421 (5.1H11, 362552, BioLegend, 1:200), anti-TCR gd Pe-Cy7 (B1, 331222, BioLegend, 1:50), anti-CD69, PerCP-Cy5.5 (FN50, 310926, BioLegend, 1:50), anti-CD161 AF488 (HP-3G10, 339924, BioLegend, 1:50), anti-NKG2D APC (1D11, 320808, BioLegend, 1:50), antiCD3 AF700 (OKT3, 317340, BioLegend, 1:200), anti-CD16 APC-Cy7 (3G8, 302018, 1:100), anti-CD14 APC-Cy7 (HCD14, 325620, 1:100). Panel 4: anti-CD4 BV785 (OKT4, 317442, BioLegend, 1:50), anti-IFN-y BV650 (4S.B3, 502538, BioLegend, 1:50), anti-CD8a BV6050 (RPA-T8, 301040, BioLegend, 1:100), anti-IL-2 BV510 (MQ1-17H12, 500338, BioLegend, 1:50), anti-CD56 BV421 (5.1H11, 362552, BioLegend, 1:200), anti-TCR gd Pe-Cy7 (B1, 331222, BioLegend, 1:50), anti-IL-10 PE-Dazzle 594 (JES3-9D7, 501426, 1:50), anti-IL-4 PE (8D8-4, 500705, BioLegend, 1:50), anti-TNF-a PerCP-Cy5.5 (MAb11, 502926, BioLegend, 1:50), anti-IL-8 FITC (E8N1, 511406, BioLegend, 1:50), anti-CD3 AF700 (OKT3, 317340, BioLegend, 1:200), anti-IL-17A AF647 (BL168, 512310, BioLegend, 1:50), anti-IL-17F AF647 (O33-782, 561333, BD, 1:50).

The following antibodies were used in SARS-CoV-2 specific antibody experiments (cat number where applicable, supplier where applicable, dilution): goat-anti-human-Fc-AP (109-055-098, Jackson, 1:1000), goat-anti-human-IgM-HRP (A6907, Sigma, 1:1000), CR3009 (2ug/ml), CR3022 (0.2ug/ml).

The following antibodies were used in the SARS-CoV-2 specific FluoroSpot experiments (clone where applicable, cat number, supplier, dilution): anti-IFN-y monoclonal capture antibody (1-D1-K, FSX-01A-1, Mabtech, 15ug/ml), anti-IFN-y+ BAM monoclonal detection antibody (7-B6-1-BAM, FSX-01A-1, Mabtech, 1:200), anti-BAM-490 (FSX-01A-1, Mabtech, 1:200).

Validation

CR3022 and CR3009 positive control antibodies (for SARS-CoV-2 antibody testing) were validated in Pickering et al PLoS Pathogens, 2020 24;16(9):e1008817. All other antibodies used in all experiments are those commonly used in immunology studies and purchased from reputable companies that have validated these antibodies. Please refer to the supplier specification sheets for further details. All flow cytometry antibodies were titrated using human adult PBMCs prior to use on our neonatal cohorts.

\section{Human research participants}

Policy information about studies involving human research participants

Population characteristics

Recruitment

Ethics oversight
Neonates $(n=30)$ born to mothers who were exposed to SARS-CoV-2 at any time during the pregnancy (and their mothers: $\mathrm{n}=28$ ) were recruited to this study during the pandemic, 28th May 2020-1st March 2021 [SE group: divided into R/O and R groups]. 28 neonates were singletons, 2 of the neonates were a twin (born to 1 mother). 1 neonate's mother's peripheral blood was not collected due to logistical reasons and are thus not included in the maternal $n$ numbers. An additional group of neonates only $(n=15)$ born to mothers who were never exposed to SARS-CoV-2 at any time during the pregnancy (before the pandemic, until 1st Jan 2020) were also recruited to this study [NSE group] as a control group for the flow cytometry work. Median maternal ages were as follows: R/O: 35, R: 32, NSE: 36. Similar distributions of male and female neonates were in our groups (Males - R/O: 9, R: 8, NSE 8 and Females - R/O: 7, R: 6, NSE: 7). Detailed maternal and neonatal characteristics including maternal history are found in Table 1 . An additional $n=5$ NSE neonates were utilised as a control group for the IFNy+ SARS-CoV-2 peptide fluorospots.

The NSE group were recruited prior to the pandemic from women booked for a planned caesarean section at GSTT and screened for non-infectious status. The SE group were also recruited from women attending GSTT maternity services and selected based on confirmed SARS-CoV-2 status prior to delivery and included both CS and NVD. Extended data fig 5 addresses any differences due to different modes of delivery. All women were asked to provided written informed consent and provide baseline samples including maternal and cord blood. Participants were not compensated for their consent.

This study was conducted in accordance with ethical approval:

SE cohort: REC Approval No. 19/SC/0232 (Infectious Diseases Bio-Bank - Guys \& St Thomas's Hospital, South Central Hampshire B REC)

NSE cohort: REC Approval No. 17/LO/0641 (London - Camberwell St Giles REC) 
Plots

Confirm that:

\The axis labels state the marker and fluorochrome used (e.g. CD4-FITC).

\The axis scales are clearly visible. Include numbers along axes only for bottom left plot of group (a 'group' is an analysis of identical markers).

\All plots are contour plots with outliers or pseudocolor plots.

$\bigotimes$ A numerical value for number of cells or percentage (with statistics) is provided.

\section{Methodology}

Sample preparation

Instrument

Software

Cell population abundance

Gating strategy
Umbilical cord blood samples that were collected in herapinised tubes (from all neonatal groups) were used in flow cytometry experiments. As indicated in the methods, blood was layered over Ficoll (GE Healthcare) and centrifuged at $800 \mathrm{x}$ g, 15 mins (break off) at room temperature to separate the cord blood mononuclear cells (CBMCs). CBMCs from all neonatal groups (R/O, R, NSE) were washed twice and were then frozen in Cryostor CS10 (Sigma) and stored in liquid nitrogen.

FCS files from cell immunophenotyping data was collected on a 4-laser LSR Fortessa (BD). FCS files from multiplex cytokine analysis were collected using a FACSCanto (BD).

Cell immunophenotyping FCS files were collected using FACSDiva (BD) and analysed on FlowJo (v10.6.2, Treestar). FCS files resulting from the multiple cytokine testing were collected using FACSDiva (BD) analysed on LEGENDplex Software for PC (v8, Biolegend).

No cells were sorted.

Gating strategies are found within the Extended Data Fig. 6. In brief, cells were cleaned up from the Time vs SSC-A gate to ensure that only cells that were through a constant flow stream were anlaysed. Live singlet cells were gated on and the resulting cell populations were then gated on as indicated in Extended Data Fig 6.

$\bigotimes$ Tick this box to confirm that a figure exemplifying the gating strategy is provided in the Supplementary Information. 